\title{
IS THE FUZZY POTTS MODEL GIBBSIAN?
}

\author{
Olle HÄGGSTRÖM ${ }^{1}$ \\ Mathematical Statistics, Chalmers University of Technology, 41296 Göteborg, Sweden
}

Received 24 April 2002, accepted 13 December 2002

\begin{abstract}
The fuzzy Potts model is obtained by looking at the Potts model with a pair of glasses that prevents distinguishing between some of the spin values. We show that the fuzzy Potts model on $\mathbf{Z}^{d}(d \geqslant 2)$ is Gibbsian at high temperatures and non-Gibbsian at low temperatures.

๑ 2003 Published by Éditions scientifiques et médicales Elsevier SAS

RÉSUMÉ. - Le modèle de Potts flou est obtenu en regardant le modèle de Potts à travers des lunettes qui ne permettent pas de distinguer certaines des valeurs de spin. Nous montrons que le modèle de Potts flou sur $\mathbf{Z}^{d}(d \geqslant 2)$ est de Gibbs à haute température mais ne l'est pas à basse température.

๑ 2003 Published by Éditions scientifiques et médicales Elsevier SAS
\end{abstract}

\section{Introduction}

This paper is concerned with the question of whether or not the so-called fuzzy Potts model is Gibbsian. Roughly speaking, the fuzzy Potts model arises by hiding some of the information about the spin variables in the usual Potts model. It provides what is possibly the simplest nontrivial example from a wider class of processes known as hidden Markov random fields [23]. These are useful in statistical image analysis and other areas of applied probability. Furthermore, the fuzzy Potts model has turned out to be a useful device in the study of percolation-theoretic properties of the Potts model; see [5] and [18]. The focus of this paper will mainly be on the fuzzy Potts model on $\mathbf{Z}^{d}$ in $d \geqslant 2$ dimensions.

Since the seminal paper by van Enter, Fernández and Sokal [8], it has been widely recognized that many random fields of physical interest fail to be Gibbsian, and much work has been put into the problem of determining whether Gibbsianness holds in various models (see, e.g., [6,7,9-11,21,22] and the references therein). For the fuzzy Potts model on $\mathbf{Z}^{d}$, this question was touched upon by Maes and Vande Velde [25], but no conclusive answer was given. Here we shall show that the answer depends on the temperature parameter: For $d \geqslant 2$, Gibbsianness holds at high but not at low temperatures,

E-mail address: olleh@math.chalmers.se (O. Häggström).

URL address: http://www.math.chalmers.se/ olleh/ (O. Häggström).

${ }^{1}$ Research supported by the Swedish Research Council. 
see Theorem 3.2. There is an intermediate regime in which we are unable to determine the answer, although in Conjecture 3.3 we make an educated guess at a precise condition.

Related to Gibbsianness is the stronger notion of $k$-Markovianness. We shall see in Theorem 7.2 that (for any $k$ and any $d \geqslant 2$ ) the fuzzy Potts model on $\mathbf{Z}^{d}$ fails to be $k$-Markovian at all temperatures.

The rest of the paper is organized as follows. In Section 2 we introduce the model. In Section 3 we recall the general notion of Gibbsianness and state the main result (Theorem 3.2). In Section 4 we observe how the question of Gibbsianness can be reduced to that of quasilocality. A key tool in proving quasilocality (and nonquasilocality) is the use of the Fortuin-Kasteleyn random-cluster representation of the Potts model, which we recall in Sections 5 and 6, and exploit in Sections 7-9 to finish the proof of the main result.

\section{The model}

In order to define the fuzzy Potts model, we first need to recall the definition of the Potts model. Although our main interest is in the case where these models live on the infinite cubic lattice $\mathbf{Z}^{d}$, we begin with the case of finite graphs.

Let $q$ be a positive integer. The $q$-state Potts model on a finite graph $G=(V, E)$ is a random assignment of $\{1, \ldots, q\}$-valued spins to the vertices of $G$. The Gibbs measure $\pi_{q, \beta}^{G}$ for the $q$-state Potts model on $G$ at inverse temperature $\beta \geqslant 0$, is the probability measure $\pi_{q, \beta}^{G}$ on $\{1, \ldots, q\}^{V}$ which to each $\xi \in\{1, \ldots, q\}^{V}$ assigns probability

$$
\pi_{q, \beta}^{G}(\xi)=\frac{1}{Z_{q, \beta}^{G}} \exp \left(2 \beta \sum_{\langle x, y\rangle \in E} I_{\{\xi(x)=\xi(y)\}}\right) .
$$

Here $\langle x, y\rangle$ denotes the edge connecting $x, y \in V, I_{A}$ is the indicator function of the event $A$, and $Z_{q, \beta}^{G}$ is a normalizing constant. This is a much-studied model in probability theory and statistical mechanics; see for instance $[13,14,17]$ and the references therein. The case $q=1$ is trivial. The Ising case $q=2$ is of course extremely interesting from various points of view, but not in the context of the fuzzy Potts model. Hence, the cases of interest in the present paper are $q=3,4, \ldots$.

Let $q$ and $\beta$ be as above, and let $s$ and $r_{1}, \ldots, r_{s}$ be positive integers such that $\sum_{i=1}^{s} r_{i}=q$. The fuzzy Potts model on $G$ with these parameters arises by taking the $q$-state Potts model on $G$, and then identifying $r_{1}$ of the Potts states with a single fuzzy spin value $1, r_{2}$ of the states with fuzzy spin value 2 , and so on. A more precise definition is as follows. Fix $q, \beta$ and $\left(r_{1}, \ldots, r_{s}\right)$ as above. Let $X$ be a $\{1, \ldots, q\}^{V}$-valued random object distributed according to the Gibbs measure $\pi_{q, \beta}^{G}$. Then take $Y$ to be the $\{1, \ldots, s\}^{V}$-valued random object obtained from $X$ by setting

$$
Y(x)=\left\{\begin{array}{cc}
1 & \text { if } X(x) \in\left\{1, \ldots, r_{1}\right\}, \\
2 & \text { if } X(x) \in\left\{r_{1}+1, \ldots, r_{1}+r_{2}\right\}, \\
\vdots & \vdots \\
s & \text { if } X(x) \in\left\{\sum_{i=1}^{s-1} r_{s}+1, \ldots, q\right\}
\end{array}\right.
$$


for each $x \in V$. We write $\mu_{q, \beta,\left(r_{1}, \ldots, r_{s}\right)}^{G}$ for the induced probability measure on $\{1, \ldots, s\}^{V}$, and call it the fuzzy Potts measure with parameters $q, \beta$, and $\left(r_{1}, \ldots, r_{s}\right)$. This definition is a slight generalization, and a unification, of definitions given by Maes and Vande Velde [25] (who considered the case $r_{1}=\cdots=r_{s}$ ), and Häggström [18] (who considered the case $s=2$ ). The case where $s=2$ and $r_{1}=1$ has also been studied by Chayes [5].

In the following, we shall assume that $1<s<q$, since the case $s=1$ is trivial, whereas the case $s=q$ just reproduces the Potts model (hence the earlier remark that we restrict attention to $q \geqslant 3$ ). It will also be convenient to assume that

$$
r_{1}=\min \left\{r \geqslant 2: r=r_{i} \text { for some } i \in\{1, \ldots, s\}\right\} .
$$

By permutation and relabeling of the sets of spin values, we see that there is no loss of generality in making such an assumption.

We move on to the infinite case $G=\mathbf{Z}^{d}$, which is shorthand notation for the graph whose vertex set $V$ is $\mathbf{Z}^{d}$, and whose edge set $E^{d}$ consists of pairs of vertices at Euclidean distance 1 from each other. For $W \subset V$, we define the boundary

$$
\partial W=\left\{x \in V \backslash W: \exists y \in W \text { such that }\langle x, y\rangle \in E^{d}\right\} .
$$

A probability measure $\pi$ on $\{1, \ldots, q\}^{\mathbf{Z}^{d}}$ is said to be a Gibbs measure for the $q$-state Potts model on $\mathbf{Z}^{d}$ at inverse temperature $\beta$, if it admits conditional probabilities such that for all finite $W \subset \mathbf{Z}^{d}$, all $\xi \in\{1, \ldots, q\}^{W}$ and all $\eta \in\{1, \ldots, q\}^{\mathbf{Z}^{d} \backslash W}$ we have

$$
\begin{aligned}
\pi & \left(X(W)=\xi \mid X\left(\mathbf{Z}^{d} \backslash W\right)=\eta\right) \\
& =\frac{1}{Z_{q, \beta}^{W, \eta}} \exp \left(2 \beta\left(\sum_{\substack{\langle x, y\rangle \in E \\
x, y \in W}} I_{\{\xi(x)=\xi(y)\}}+\sum_{\substack{\langle x, y\rangle \in E \\
x \in W, y \in \partial W}} I_{\{\xi(x)=\eta(y)\}}\right)\right),
\end{aligned}
$$

where the normalizing constant $Z_{q, \beta}^{W, \eta}$ is allowed to depend on $\eta$ but not on $\xi$.

The basic examples of Gibbs measures for the Potts model are constructed as follows. Let $\Lambda_{1} \subset \Lambda_{2} \subset \cdots$ be a sequence of finite vertex sets converging to $\mathbf{Z}^{d}$ in the sense that each $x \in \mathbf{Z}^{d}$ is in all but finitely many $\Lambda_{n}$ 's. Let $G_{n}$ denote the graph whose vertex set is $\Lambda_{n} \cup \partial \Lambda_{n}$, and whose edge set consists of pairs of vertices in $\Lambda_{n} \cup \partial \Lambda_{n}$ at Euclidean distance 1 from each other. It is well known (see, e.g., [17] or [14] for this and other results quoted in this section) that the Gibbs measures $\pi_{q, \beta}^{G_{n}}$ converge to a probability measure on $\{1, \ldots, q\}^{\mathbf{Z}^{d}}$ which is a Gibbs measure for the Potts model on $\mathbf{Z}^{d}$ with the given parameters. Convergence takes place in the sense that probabilities of cylinder sets converge. The limiting measure is denoted $\pi_{q, \beta}^{\mathbf{Z}^{d}, 0}$, and is called the Gibbs measure (for the Potts model on $\mathbf{Z}^{d}$ with the given parameters) with free boundary condition. Other Gibbs measures are those with so-called spin $i$ boundary condition, denoted $\pi_{q, \beta}^{\mathbf{Z}^{d}, i}$, for $i=1, \ldots, q$. These are obtained by conditioning $\pi_{q, \beta}^{G_{n}}$ on taking spin value $i$ all over $\partial \Lambda_{n}$ - yielding another probability measure $\pi_{q, \beta}^{G_{n, i}}$ on $\{1, \ldots, q\}^{\Lambda_{n} \cup \partial \Lambda_{n}}$ - and then taking limits as $n \rightarrow \infty$. Each of the measures $\pi_{q, \beta}^{\mathbf{Z}^{d}, 0}, \ldots, \pi_{q, \beta}^{\mathbf{Z}^{d}, q}$ is independent of the particular choice of $\left\{\Lambda_{n}\right\}_{n=1}^{\infty}$. 
In dimension $d \geqslant 2$, these Gibbs measures may or may not coincide depending on the parameter values. It is a fundamental result due to Aizenman et al. [1], that there exists a critical value $\beta_{c}=\beta_{c}(d, q) \in(0, \infty)$, such that for $\beta<\beta_{c}$, there is only one Gibbs measure (so that in particular $\pi_{q, \beta}^{\mathbf{Z}^{d}, 0}=\cdots=\pi_{q, \beta}^{\mathbf{Z}^{d}, q}$ ), whereas for $\beta>\beta_{c}$, there are multiple Gibbs measures (moreover, the measures $\pi_{q, \beta}^{\mathbf{Z}^{d}, 0}, \ldots, \pi_{q, \beta}^{\mathbf{Z}^{d}, q}$ are all distinct).

For $q, \beta$, and $\left(r_{1}, \ldots, r_{s}\right)$ as above, and $i=0, \ldots, q$, we define the fuzzy Potts measure $\mu_{q, \beta,\left(r_{1}, \ldots, r_{s}\right)}^{\mathbf{Z}^{d}, i}$ to be the distribution of the $\{1, \ldots, s\} \mathbf{Z}^{d}$-valued random object $Y$ obtained by first picking $X \in\{1, \ldots, q\}^{\mathbf{Z}^{d}}$ according to the Gibbs measure $\pi_{q, \beta}^{\mathbf{Z}^{d}, i}$, and then constructing $Y$ from $X$ as in (2).

\section{The main result}

In order to state our main result regarding (non-)Gibbsianness of the fuzzy Potts model, we need to recall the general notion of a Gibbs measure. For probability measures on $S^{\mathbf{Z}^{d}}$ in the case where the single site state space $S=\left\{s_{1}, \ldots, s_{k}\right\}$ is finite, it amounts to the following; see [13] or [8] for more detail.

Let $\mathcal{W}$ be the class of all finite subsets of $\mathbf{Z}^{d}$. An interaction potential is a family $\Phi=\left\{\Phi_{W}\right\}_{W \in \mathcal{W}}$ of functions $\Phi_{W}: S^{\mathbf{Z}^{d}} \rightarrow \mathbf{R}$ with the property that $\Phi_{W}(\xi)=\Phi_{W}\left(\xi^{\prime}\right)$ for all $\xi, \xi^{\prime} \in S^{\mathbf{Z}^{d}}$ such that $\xi(x)=\xi^{\prime}(x)$ for all $x \in W$. In other words, the function $\Phi_{W}$ is only allowed to depend on the spins in the finite subset $W$. For $W \in \mathcal{W}$, define

$$
\left\|\Phi_{W}\right\|=\max _{\xi, \xi^{\prime} \in S^{Z^{d}}}\left|\Phi_{W}(\xi)-\Phi_{W}\left(\xi^{\prime}\right)\right| .
$$

The interaction potential $\Phi$ is said to be absolutely summable if for all $x \in \mathbf{Z}^{d}$ we have

$$
\sum_{W \ni x}\left\|\Phi_{W}\right\|<\infty .
$$

DEFINITION 3.1. - Suppose that $\Phi$ is absolutely summable. A probability measure $\mu$ on $S^{\mathbf{Z}^{d}}$ is said to be a Gibbs measure for the interaction potential $\Phi$ if it admits conditional probabilities such that for all $x \in \mathbf{Z}^{d}$, all $s, s^{\prime} \in S$ and all $\eta \in S^{\mathbf{Z}^{d} \backslash\{x\}}$ we have

$$
\frac{\mu\left(X(x)=s \mid X\left(\mathbf{Z}^{d} \backslash\{x\}\right)=\eta\right)}{\mu\left(X(x)=s^{\prime} \mid X\left(\mathbf{Z}^{d} \backslash\{x\}\right)=\eta\right)}=\exp \left(\sum_{\substack{W \in \mathcal{W} \\ W \ni x}}\left(\Phi_{W}\left(\eta \vee s^{\prime}\right)-\Phi_{W}(\eta \vee s)\right)\right)
$$

where $(\eta \vee s) \in S^{\mathbf{Z}^{d}}$ is the configuration which agrees with $\eta$ on $\mathbf{Z}^{d} \backslash\{x\}$ and which takes value s on $x$. More generally, we say that $\mu$ is a Gibbs measure if it is a Gibbs measure for some absolutely summable interaction potential.

Recall from Section 2 that $\beta_{c}(d, q)$ denotes the critical inverse temperature for the $q$-state Potts model on $\mathbf{Z}^{d}$.

One more definition is needed to formulate our main result: Let $p_{c}(d)$ denote the critical value for i.i.d. bond percolation on $\mathbf{Z}^{d}$. That is, $p_{c}(d)$ is the supremum of the 
set of $p \in[0,1]$ such that if each edge in $\mathbf{Z}^{d}$ is removed independently with probability $1-p$, then a.s. all remaining connected components are finite. For $d \geqslant 2$, we have $p_{c}(d) \in(0,1)$; see [16] for this and much more on percolation.

Recall also the convention (3) concerning the parameter $r_{1}$ in $\mu_{q, \beta,\left(r_{1}, \ldots, r_{s}\right)}^{\mathbf{Z}^{d}, i}$.

THeorem 3.2. - Consider the fuzzy Potts measure $\mu_{q, \beta,\left(r_{1}, \ldots, r_{s}\right)}^{\mathbf{Z}^{d, i}}$ with $d \geqslant 2, q \geqslant 3$, fixed $i \in\{0, \ldots, q\}$, and $\left(r_{1}, \ldots, r_{s}\right)$ satisfying $1<s<q$.

(i) For $\beta<\beta_{c}\left(d, r_{1}\right)$, the measure $\mu_{q, \beta,\left(r_{1}, \ldots, r_{s}\right)}^{\mathbf{Z}^{d}, i}$ is a Gibbs measure.

(ii) In contrast, for $\beta>\frac{1}{2} \log \left(\left(1+\left(r_{1}-1\right) p_{c}(d)\right) /\left(1-p_{c}(d)\right)\right), \mu_{q, \beta,\left(r_{1}, \ldots, r_{s}\right)}^{\mathbf{Z}^{d}, i}$ is not a Gibbs measure.

After some preliminaries in Sections 4-7, we will prove this result in Sections 8 and 9. In Section 8 we will also give an incomplete argument in support of the following.

CONJECTURE 3.3. - Theorem 3.2(i) is sharp in the sense that $\mu_{q, \beta,\left(r_{1}, \ldots, r_{s}\right)}^{\mathbf{Z}^{d}, i}$ is nonGibbsian for all $\beta>\beta_{c}\left(d, r_{1}\right)$.

Maes and Vande Velde [25] conjectured (in the symmetric setting where $r_{1}=\cdots=r_{s}$ ) that $\mu_{q, \beta,\left(r_{1}, \ldots, r_{s}\right)}^{\mathbf{Z}^{d}, i}$ is non-Gibbsian for $\beta>\beta_{c}(q, d)$, i.e., in the nonuniqueness regime for the underlying Potts model. Although Theorem 3.2(ii) does not prove the full MaesVande Velde conjecture, it does go further in certain parts of the parameter space. To see this, we recall the well-known result of Laanait, Messager and Ruiz [24] that for the the $q$-state Potts model on $\mathbf{Z}^{2}$, the critical value satisfies $\beta_{c}(q, 2)=\frac{1}{2} \log (\sqrt{q}+1)$ for $q$ large enough ( $q>25$ suffices). By taking $d=2$, fixing $r_{1}$, and taking $q$ to be sufficiently large, we see that Theorem 3.2(ii) in conjunction with the Laanait-Messager-Ruiz result implies that the fuzzy Potts measure can be non-Gibbsian even in the absence of phase transition (multiple Gibbs measures) in the underlying Potts model. This phenomenon (non-Gibbsianness in the absence of phase transition) arises as a consequence of the fact that reducing the number of spin states $q$ in the Potts model decreases the critical value $\beta_{c}(d, q)$, similarly as in the non-Gibbsianness phenomena discussed by van Enter, Fernández and Kotecký [7].

\section{Gibbsianness and quasilocality}

The purpose of this section is to reduce the issue of Gibbsianness for fuzzy Potts measures, to that of so-called quasilocality for the same measures. Loosely speaking, a measure $\mu$ on $S^{\mathbf{Z}^{d}}$ is quasilocal if a random spin configuration $X$ with distribution $\mu$ has the property that for finite $W \subset \mathbf{Z}^{d}$, the conditional distribution of $X(W)$ given $X\left(\mathbf{Z}^{d} \backslash W\right)$ does not depend strongly on spins very far away.

As in Section 2, we fix a sequence $\Lambda_{1} \subset \Lambda_{2} \subset \cdots$ of finite vertex sets converging to $\mathbf{Z}^{d}$. The following definition is independent of the particular choice of $\left\{\Lambda_{n}\right\}_{n=1}^{\infty}$.

DEFINITION 4.1. - Let $S=\left\{s_{1}, \ldots, s_{k}\right\}$ be a finite set. A probability measure $\mu$ on $S^{\mathbf{Z}^{d}}$ is said to be quasilocal if it admits conditional probabilities such that for all $W \in \mathcal{W}$ and all $\xi \in S^{W}$ we have 


$$
\begin{array}{ll}
\lim _{n \rightarrow \infty} \sup _{\substack{\eta, \eta^{\prime} \in S^{\mathbf{Z}^{d} \backslash W} \\
\eta\left(\Lambda_{n} \backslash W\right)=\eta^{\prime}\left(\Lambda_{n} \backslash W\right)}} \mid \mu\left(X(W)=\xi \mid X\left(\mathbf{Z}^{d} \backslash W\right)=\eta\right) \\
& -\mu\left(X(W)=\xi \mid X\left(\mathbf{Z}^{d} \backslash W\right)=\eta^{\prime}\right) \mid=0 .
\end{array}
$$

Another relevant concept is that of uniform nonnullness; readers familiar with the socalled finite energy [26] condition will find that uniform nonnullness is a strong form of finite energy.

DEFINITION 4.2. - Let $S=\left\{s_{1}, \ldots, s_{k}\right\}$ be a finite set. A probability measure $\mu$ on $S^{\mathbf{Z}^{d}}$ is said to be uniformly nonnull if for some $\varepsilon>0$ it admits conditional probabilities such that for all $x \in \mathbf{Z}^{d}$, all $s \in S$ and all $\eta \in S^{\mathbf{Z}^{d} \backslash\{x\}}$ we have

$$
\mu\left(X(x)=s \mid X\left(\mathbf{Z}^{d} \backslash\{x\}\right)=\eta\right) \geqslant \varepsilon .
$$

The following characterization of Gibbsianness is well known; see, e.g., [8, Theorem 2.12].

THEOREM 4.3. - A probability measure $\mu$ on $S^{\mathbf{Z}^{d}}$, where $S$ is a finite set, is a Gibbs measure if and only if it is quasilocal and uniformly nonnull.

Our main result (Theorem 3.2) is therefore established as soon as we have proved the following two results.

THEOREM 4.4. - Consider the fuzzy Potts measure $\mu_{q, \beta,\left(r_{1}, \ldots, r_{s}\right)}^{\mathbf{Z}^{d}, i}$ with $d \geqslant 2$ and parameter values as in Theorem 3.2.

(i) For $\beta<\beta_{c}\left(d, r_{1}\right), \mu_{q, \beta,\left(r_{1}, \ldots, r_{s}\right)}^{\mathbf{Z}^{d}, i}$ is quasilocal.

(ii) For $\beta>\frac{1}{2} \log \left(\left(1+\left(r_{1}-1\right) p_{c}(d)\right) /\left(1-p_{c}(d)\right)\right), \mu_{q, \beta,\left(r_{1}, \ldots, r_{s}\right)}^{\mathbf{Z}^{d}, i}$ is not quasilocal.

LEMMA 4.5. - The fuzzy Potts measure $\mu_{q, \beta,\left(r_{1}, \ldots, r_{s}\right)}^{\mathbf{Z}^{d}, i}$ with arbitrary parameter values, is uniformly nonnull.

Our main task is to prove Theorem 4.4; this is deferred to Sections 8 and 9. Lemma 4.5, on the other hand, turns out to be easy.

Proof of Lemma 4.5. - Take the Potts configuration $X \in\{1, \ldots, q\}^{\mathbf{Z}^{d}}$ to have distribution $\pi_{q, \beta}^{\mathbf{Z}^{d}, i}$, and let $Y \in\{1, \ldots, s\}^{\mathbf{Z}^{d}}$ be its corresponding fuzzy Potts configuration. $Y$ then has distribution $\mu_{q, \beta,\left(r_{1}, \ldots, r_{s}\right)}^{\mathbf{Z}^{d}, i}$. A direct calculation using (4) yields that for any $x \in \mathbf{Z}^{d}$, any $a \in\{1, \ldots, q\}$ and any $\xi \in\{1, \ldots, q\}^{\mathbf{Z}^{d} \backslash\{x\}}$ we have

$$
\mathbf{P}\left(X(x)=a \mid X\left(\mathbf{Z}^{d} \backslash\{x\}\right)-\xi\right) \geqslant \frac{1}{q-1+\mathrm{e}^{4 d \beta}} .
$$

Hence, for any $b \in\{1, \ldots, s\}$ we have

$$
\mathbf{P}\left(Y(x)=b \mid X\left(\mathbf{Z}^{d} \backslash\{x\}\right)=\xi\right) \geqslant \frac{r_{b}}{q-1+\mathrm{e}^{4 d \beta}} .
$$


Note now that for any $\eta \in\{1, \ldots, s\}^{\mathbf{Z}^{d} \backslash\{x\}}$, the expression $\mathbf{P}\left(Y(x)=b \mid Y\left(\mathbf{Z}^{d} \backslash\{x\}\right)=\eta\right)$ is a convex combination of terms of the form of the left-hand side of (6). Hence

$$
\mathbf{P}\left(Y(x)=b \mid Y\left(\mathbf{Z}^{d} \backslash\{x\}\right)=\eta\right) \geqslant \frac{r_{b}}{q-1+\mathrm{e}^{4 d \beta}}
$$

so that $\mu_{q, \beta,\left(r_{1}, \ldots, r_{s}\right)}^{\mathbf{Z}^{d}, i}$ is uniformly nonnull with

$$
\varepsilon=\frac{\min _{b \in\{1, \ldots, s\}} r_{b}}{q-1+\mathrm{e}^{4 d \beta}}
$$

\section{The random-cluster representation in finite volume}

The random-cluster model of Fortuin and Kasteleyn [12] has been a major tool in the study of Ising and Potts models ever since the publication of the influential papers by Swendsen and Wang [28] and Aizenman et al. [1]. See [17] and [14] for surveys of such work. Not surprisingly, the random-cluster model is useful also in the analysis of the fuzzy Potts model, as witnessed in [5,25], and [18]. In this section and the next, we recall the random-cluster model and discuss its relation to the fuzzy Potts model. The simpler case of finite graphs is treated in this section, while the infinite-volume case is handled in Section 6.

Let $G=(V, E)$ be a finite graph. For $p \in[0,1]$ and $q>0$, we define the randomcluster measure $\phi_{p, q}^{G}$ on $\{0,1\}^{E}$ as the probability measure which to each edge configuration $\xi \in\{0,1\}^{E}$ assigns probability

$$
\phi_{p, q}^{G}(\xi)=\frac{q^{k(\xi)}}{\widehat{Z}_{p, q}^{G}} \prod_{e \in E} p^{\xi(e)}(1-p)^{(1-\xi(e))} .
$$

Here $k(\xi)$ is the number of connected components of the graph with vertex set $V$ and edge set $\{e \in E: \xi(e)=1\}$, and $\widehat{Z}_{p, q}^{G}$ is a normalizing constant. We generally think of edges $e$ with $\xi(e)=0$ as deleted, and those with $\xi(e)=1$ as retained. Taking $q=1$ yields i.i.d. measure with retention probability $p$, whereas $q>1$ (resp. $q<1$ ) biases the measure in favor of configurations with many (resp. few) connected components.

The well-known correspondence between random-cluster and Potts models may be phrased as follows. Pick a random edge configuration $U \in\{0,1\}^{E}$ according to the random-cluster measure $\phi_{p, q}^{G}$ with $q \in\{2,3, \ldots\}$. Then pick a spin configuration $X \in$ $\{1, \ldots, q\}^{V}$ by letting each connected component of $U$ receive the same spin value on all its vertices, chosen uniformly from $\{1, \ldots, q\}$, and doing this independently for each connected component. It turns out that the resulting spin configuration $X$ is then distributed according to the Gibbs measure $\pi_{q, \beta}^{G}$ for the $q$-state Potts model with $\beta=-\frac{1}{2} \log (1-p)$.

For positive integers $r_{1}, \ldots, r_{s}$ such that $\sum_{i=1}^{s} r_{i}=q$, we can of course go on and construct $Y \in\{1, \ldots, s\}^{V}$ from $X$ using (2), and $Y$ will then be distributed according to the fuzzy Potts measure $\mu_{q, \beta,\left(r_{1}, \ldots, r_{s}\right.}^{G}$. But instead of going via the Potts model, we may as well obtain $Y$ directly from the random-cluster model by assigning spins from $\{1, \ldots, s\}$ 
to the connected components of $U$, with probability $r_{i} / q$ of getting spin value $i$, and independently for different components. Of crucial importance to us in proving our main result will be to understand the conditional distribution of the edge configuration $U$ given the fuzzy spin configuration $Y$. The following result generalizes results in [18] and in [25].

PROPOSITION 5.1. - For given $p, q,\left(r_{i}, \ldots, r_{s}\right)$ and a finite graph $G=(V, E)$, pick a random edge configuration $U \in\{0,1\}^{E}$ and a fuzzy spin configuration $Y \in\{1, \ldots, s\}^{V}$ as above. For any $\eta \in\{1, \ldots, s\}^{V}$, the conditional distribution of $U$ given $Y=\eta$ is as follows.

For $i=1, \ldots, s$, define $G^{\eta, i}=\left(V^{\eta, i}, E^{\eta, i}\right)$ as the graph with vertex set $V^{\eta, i}=\{v \in$ $V: \eta(v)=i\}$ and edge set $E^{\eta, i}=\left\{e \in E:\right.$ both endpoints of $e$ are in $\left.V^{\eta, i}\right\}$. Independently for each $i, U\left(E^{\eta, i}\right)$ is distributed according to the random-cluster measure $\phi_{p, r_{i}}^{G^{\eta, i}}$. Finally, any edge e in $E \backslash\left(\bigcup_{i=1}^{s} E^{\eta, i}\right)$ takes value $U(e)=0$ with probability 1.

Proof. - Fix $\eta \in\{1, \ldots, s\}^{V}$, and suppose that $Y=\eta$. That $U(e)=0$ for any $e \in$ $E \backslash\left(\bigcup_{i=1}^{s} E^{\eta, i}\right)$ is immediate from the construction of $Y$. Now fix $\xi \in\{0,1\}^{E}$ such that $\xi(e)=0$ for all $e \in E \backslash\left(\bigcup_{i=1}^{s} E^{\eta, i}\right)$. Write $n(\eta)$ for the number of edges in $E$ whose endpoints take different values in $\eta$. Furthermore, for $i=1, \ldots, s$, let $k_{i}(\xi, \eta)$ denote the number of connected components of $\xi$ that receive fuzzy spin $i$ in $\eta$. We get that

$$
\begin{aligned}
\mathbf{P}(U=\xi, Y=\eta) & =\phi_{p, q}^{G}(\xi) \prod_{i=1}^{s}\left(\frac{r_{i}}{q}\right)^{k_{i}(\xi, \eta)} \\
& =\frac{q^{k(\xi)}}{\widehat{Z}_{p, q}^{G}} \prod_{e \in E} p^{\xi(e)}(1-p)^{(1-\xi(e))} \prod_{i=1}^{s}\left(\frac{r_{i}}{q}\right)^{k_{i}(\xi, \eta)} \\
& =\frac{(1-p)^{n(\eta)}}{\widehat{Z}_{p, q}^{G}} \prod_{i=1}^{s}\left(r_{i}^{k_{i}(\xi, \eta)} \prod_{e \in E^{\eta, i}} p^{\xi(e)}(1-p)^{(1-\xi(e))}\right),
\end{aligned}
$$

where the last equality uses that $\sum_{i=1}^{s} k_{i}(\xi, \eta)=k(\xi)$ and is otherwise just a rearrangement of factors. Hence,

$$
\begin{aligned}
\mathbf{P}(U=\xi \mid Y=\eta) & =\frac{\mathbf{P}(U=\xi, Y=\eta)}{\mathbf{P}(Y=\eta)} \\
& =\frac{(1-p)^{n(\eta)}}{\widehat{Z}_{p, q}^{G} \mathbf{P}(Y=\eta)} \prod_{i=1}^{s}\left(r_{i}^{k_{i}(\xi, \eta)} \prod_{e \in E^{\eta, i}} p^{\xi(e)}(1-p)^{(1-\xi(e))}\right) \\
& =\frac{(1-p)^{n(\eta)} \prod_{i=1}^{s} \widehat{Z}_{p, r_{i}}^{G^{\eta, i}} \prod_{i=1}^{s} \phi_{p, r_{i}}^{G^{\eta, i}}\left(\xi\left(E^{\eta, i}\right)\right)}{\widehat{Z}_{p, q}^{G} \mathbf{P}(Y=\eta)} \\
& =\prod_{i=1}^{s} \phi_{p, r_{i}}^{G^{\eta, i}}\left(\xi\left(E^{\eta, i}\right)\right)
\end{aligned}
$$

because the expression that was cancelled in the last line must equal 1 by normalization.

Remark 5.2. - When a graph $G$ is not connected, it is easy to see that the randomcluster measure $\phi_{p, q}^{G}$ factorizes into $\prod_{i=1}^{n} \phi_{p, q}^{G_{i}}$, where $G_{1}, \ldots, G_{n}$ are the connected 
components of $G$. By combining this observation with Proposition 5.1, we get, for the conditional distribution of the edge configuration $U$ given the fuzzy spin configuration $Y$, the following. Partition the graph into maximal connected components such that each component is monochromatic (i.e., all vertices of the component have the same fuzzy spin). Then each such connected component $C$ has, independently of the others, an edge configuration chosen according to $\phi_{p, r_{i}}^{C}$, where $i$ is the fuzzy spin assigned to $C$.

\section{The random-cluster representation in infinite volume}

The finite-graph definition (7) of random-cluster measures cannot be applied directly to the infinite $\mathbf{Z}^{d}$ lattice, for which we instead employ the following definition.

Definition 6.1. - Let $G=(V, E)$ be an infinite, locally finite graph. A probability measure $\phi$ on $\{0,1\}^{E}$ is said to be a random-cluster measure for $G$ with parameters $p \in[0,1]$ and $q>0$ if it admits conditional probabilities such that, for $a\{0,1\}^{E}$. valued random object $U$ with distribution $\phi$, we have for any $e=\langle x, y\rangle \in E$ and any $\xi \in\{0,1\}^{E \backslash\{e\}}$ that

$$
\phi(U(e)=1 \mid U(E \backslash\{e\})=\xi)= \begin{cases}p & \text { if } x \stackrel{\xi}{\leftrightarrow} y, \\ \frac{p}{p+(1-p) q} & \text { otherwise. }\end{cases}
$$

Here $x \stackrel{\xi}{\leftrightarrow} y$ denotes the existence of a path from $x$ to $y$ consisting of edges that all take value 1 in $\xi$.

Note that the conditional probability in (8) is the same as what we get for finite graphs using the definition (7). The definition of random-cluster measures for $\mathbf{Z}^{d}$ and other infinite graphs is more often given in terms of conditional distributions on arbitrary finite edge sets, but it is well known (see, e.g., [14, Lemma 6.18]) that the above single-edge criterion is equivalent to the usual definition.

Random-cluster measures for $\mathbf{Z}^{d}$ with given parameter values can (provided that $q \geqslant 1$, which is the only case we need) be constructed as limits of random-cluster measures for finite graphs. To this end, we recall some notation from Section 2: $\Lambda_{1} \subset \Lambda_{2} \subset \cdots$ is an increasing sequence of finite vertex sets, and for each $n$ the graph $G_{n}=\left(V_{n}, E_{n}\right)$ is defined to have vertex set $V_{n}=\Lambda_{n} \cup \partial \Lambda_{n}$ and edge set $E_{n}=\left\{e \in E^{d}\right.$ : both endpoints of $e$ are in $\left.V_{n}\right\}$. In Section 2, the sequence $\left\{\Lambda_{n}\right\}$ was arbitrary, but here we have reason to specify it as $\Lambda_{n}=\{-n, \ldots, n\}^{d}$, the point being that this makes sure that $\partial \Lambda_{n}$ is connected.

For $q \geqslant 1$, it turns out (see, e.g., [17] or [14] for these and other results quoted without reference in the next few paragraphs) that the finite-volume random-cluster measures $\phi_{p, g}^{G_{n}}$ converge to a limiting measure $\phi_{p, q}^{\mathbf{Z}^{d}, \text { free }}$ on $\{0,1\}^{E^{d}}$; "free" denotes that no boundary conditions are imposed at the finite-volume stage of the construction. The limiting measure $\phi_{p, q}^{\mathbf{Z}^{d}}$,free is a random-cluster measure for $\mathbf{Z}^{d}$ with parameters $p$ and $q$, in the sense of Definition 6.1. 
There is another random-cluster measure $\phi_{p, q}^{\mathbf{Z}^{d}, \text { wired }}$ for $\mathbf{Z}^{d}$, which arises from a slight modification of this limiting procedure. Namely, let $\phi_{p, q}^{G_{n} \text {, wired }}$ denote $\phi_{p, q}^{G_{n}}$ conditioned on the event that all edges in $\left\{\langle x, y\rangle \in E_{n}: x, y \in \partial \Lambda_{n}\right\}$ take value 1 . Then $\phi_{p, q}^{\mathbf{Z}^{d} \text {,wired }}$ is the limit as $n \rightarrow \infty$ of $\phi_{p, q}^{G_{n} \text {,wired }}$ and is a random-cluster measure for $\mathbf{Z}^{d}$ with the given parameter values. The measures $\phi_{p, q}^{\mathbf{Z}^{d}, \text { free }}$ and $\phi_{p, q}^{\mathbf{Z}^{d}, \text { wired }}$ usually coincide; it is believed that the only exception is when $q$ is large and $p$ equals a certain critical value $p_{c}=p_{c}(d, q)$ which for integer $q$ satisfies $p_{c}=1-\mathrm{e}^{-2 \beta_{c}}$, where $\beta_{c}$ is the Potts model critical value defined in Section 2.

We know from Section 5 that, for integer $q$, we obtain the Gibbs measure $\pi_{q, \beta}^{G_{n}}$ for the $q$-state Potts model with $\beta=-\frac{1}{2} \log (1-p)$ from $\phi_{p, q}^{G_{n}}$ by assigning random spins (uniformly distributed on $\{1, \ldots, q\}$ ) to the connected components of the edge configuration. When the same thing is done for a random $\{0,1\}^{E_{d}}$-valued edge configuration chosen according to $\phi_{p, q}^{\mathbf{Z}^{d}, \text { free }}$, what we get is the Gibbs measure $\pi_{q, \beta}^{\mathbf{Z}^{d}, 0}$. Moreover, the joint distribution of the edge and spin variables under this procedure for finite $n$, converges as $n \rightarrow \infty$ to the joint distribution of the edge and spin variables in the infinite setting.

A similar statement is true for wired random-cluster measures: Fix $i \in\{1, \ldots, q\}$. Suppose that we pick an edge configuration according to $\phi_{p, q}^{G_{n}, \text { wired }}$ and then assign spin values to the connected components in the usual way, except that we insist on assigning spin $i$ to the connected component containing $\partial \Lambda_{n}$. Then the spin variables are distributed according to the Potts model Gibbs measure $\pi_{p, q}^{G_{n}, i}$ with $\beta=-\frac{1}{2} \log (1-p)$. The joint distribution of the edge and spin configurations converges as $n \rightarrow \infty$, with marginals tending to $\phi_{p, q}^{\mathbf{Z}^{d}, \text { wired }}$ and $\pi_{q, \beta}^{\mathbf{Z}^{d}, i}$. To go directly from the edge configuration to the spin configuration in the infinite-volume limit, we assign spins to the connected components in the usual way, except that any infinite connected component is forced to take spin value $i$.

These results about convergence of the joint distribution of edge and spin configurations are also true for the joint distribution of edge and fuzzy spin configurations; this is an immediate consequence using just the fact that the fuzzy spins are functions of the spins. The following result is our infinite-volume analogue of Proposition 5.1.

THEOREM 6.2. - Fix parameters $q \in\{3,4, \ldots\}, s \in\{2, \ldots, q-1\},\left(r_{1}, r_{2}, \ldots, r_{s}\right)$, $i \in\{0, \ldots, q\}$ and $\beta>0$ for the fuzzy Potts model on $\mathbf{Z}^{d}$, and let $p=1-\mathrm{e}^{-2 \beta}$. Suppose that we pick an edge configuration $U \in\{0,1\}^{E^{d}}$ according to $\phi_{p, q}^{\mathbf{Z}^{d}, \text { free }}$ (if $i=0$ ) or $\phi_{p, q}^{\mathbf{Z}^{d} \text {, wired }}$ (if $i>0$ ), and then a spin configuration $X \in\{1, \ldots, q\}^{\mathbf{Z}^{d}}$ by assigning spins to the connected components as described above, and finally a fuzzy spin configuration $Y \in\{1, \ldots, s\}^{\mathbf{Z}^{d}}$ as in (2). Write $\mathbf{P}$ for the joint distribution of $(U, X, Y)$. For $j=1, \ldots, s$, define $G^{Y, j}=\left(V^{Y, j}, E^{Y, j}\right)$ as the (random) graph with vertex set $V^{Y, j}=\left\{v \in \mathbf{Z}^{d}: Y(v)=j\right\}$ and edge set $E^{Y, j}=\left\{e \in E^{d}:\right.$ both endpoints of $e$ are in $\left.V^{Y, j}\right\}$.

Then $\mathbf{P}$ admits conditional probabilities such that for each $j$, the conditional distribution of $U\left(E^{Y, j}\right)$ given the full fuzzy spin configuration $Y$, is a random-cluster measure for $G^{Y, j}$ with parameters $p$ and $r_{j}$. 
It is known from [15] that $\phi_{p, q}^{\mathbf{Z}^{d}, \text { free }}$ and $\phi_{p, q}^{\mathbf{Z}^{d}, \text { wired }}$ both assign probability 1 to the existence of at most one infinite cluster. In our proof of Theorem 6.2, we shall exploit that result similarly to how Grimmett did in [15, proof of Theorem 3.1(b)].

In the proof, we will also need to consider probability measures $\mathbf{P}_{1}, \mathbf{P}_{2}, \ldots$, which are defined, similarly as $\mathbf{P}$, as the joint distribution of the edge-and-spin configuration $(U, X, Y)$, except that $\mathbf{P}_{n}$ is the $n$th finite stage of the infinite-volume construction. This means that the graph for which $\mathbf{P}_{n}$ is defined is $G_{n}$, and the marginal distribution of $X$ is

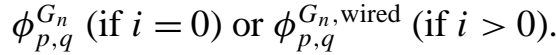

Proof of Theorem 6.2. - By the definition of random-cluster measures, we are done if we can show that $\mathbf{P}$ admits conditional probabilities such that for each $\eta \in\{1, \ldots, s\}^{\mathbf{Z}^{d}}$, each $j \in\{1, \ldots, s\}$, each $e=\langle x, y\rangle \in E$ such that $\eta(x)=\eta(y)=j$, and each $\xi \in$

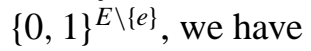

$$
\mathbf{P}(U(e)=1 \mid Y=\eta, U(E \backslash\{e\})=\xi)= \begin{cases}p & \text { if } x \stackrel{\xi}{\leftrightarrow} y, \\ \frac{p}{p+(1-p) r_{j}} & \text { otherwise. }\end{cases}
$$

For this, it is enough to show that

$$
\begin{aligned}
& \lim _{n \rightarrow \infty} \mathbf{P}\left(U(e)=1 \mid Y\left(V_{n}\right)=\eta\left(V_{n}\right), U(E \backslash\{e\})=\xi\left(E_{n} \backslash\{e\}\right)\right) \\
& \quad= \begin{cases}p & \text { if } x \stackrel{\xi}{\leftrightarrow} y, \\
\frac{p}{p+(1-p) r_{j}} & \text { otherwise }\end{cases}
\end{aligned}
$$

for a set of configurations $(\eta, \xi)$ that has $\mathbf{P}$-probability 1 .

Call an edge configuration $\xi^{\prime} \in\{0,1\}^{E^{d}}$ good if it contains at most one infinite cluster of open edges and this property is preserved under flip of any one edge-variable. By [15, Lemma 3.4], we know that the set of good edge configurations has full measure under either of $\phi_{p, q}^{\mathbf{Z}^{d}, \text { free }}$ or $\phi_{p, q}^{\mathbf{Z}^{d}, \text { wired }}$. Furthermore, if $\xi^{\prime}$ is good, then, for any $e=\langle x, y\rangle \in E$, we have either that

(i) $x$ and $y$ are in the same cluster of $\xi^{\prime}\left(E^{d} \backslash\{e\}\right)$, or that

(ii) $x$ and $y$ are in different clusters of $\xi^{\prime}\left(E^{d} \backslash\{e\}\right)$, at least one of which is finite.

Now, if (i) holds, then this can be read off from $\xi^{\prime}\left(E_{m}\right)$ for some $m$; just take $m$ to be large enough so that $x \stackrel{\xi^{\prime}\left(E_{n} \backslash\{e\}\right)}{\longleftrightarrow} y$. Let $\eta^{\prime} \in\{1, \ldots, s\}^{\mathbf{Z}^{d}}$ be any fuzzy spin configuration that is consistent with $\xi^{\prime}$. For all $n \geqslant m$ and all $k>n$, we then obtain

$$
\mathbf{P}_{k}\left(U(e)=1 \mid Y\left(V_{n}\right)=\eta^{\prime}\left(V_{n}\right), U\left(E_{n} \backslash\{e\}\right)=\xi^{\prime}\left(E_{n} \backslash\{e\}\right)\right)=p
$$

by an application of Proposition 5.1. By sending $k \rightarrow \infty$, we obtain

$$
\mathbf{P}\left(U(e)=1 \mid Y\left(V_{n}\right)=\eta^{\prime}\left(V_{n}\right), U\left(E_{n} \backslash\{e\}\right)=\xi^{\prime}\left(E_{n} \backslash\{e\}\right)\right)=p .
$$

Similarly, if (ii) holds, then there is an $m$ such that (ii) can be concluded from $\xi^{\prime}\left(E_{m}\right)$. To see this, just take $m$ large enough so that at least one of the $\xi^{\prime}$-clusters containing $x$ 
or $y$ is contained in $G_{m-1}$. Provided that $\eta^{\prime}(x)=\eta^{\prime}(y)=j$, we then get for all $n \geqslant m$ and all $k>n$ that

$$
\mathbf{P}_{k}\left(U(e)=1 \mid Y\left(V_{n}\right)=\eta^{\prime}\left(V_{n}\right), U\left(E_{n} \backslash\{e\}\right)=\xi^{\prime}\left(E_{n} \backslash\{e\}\right)\right)=\frac{p}{p+(1-p) r_{j}}
$$

by another application of Proposition 5.1. Sending $k \rightarrow \infty$ yields

$$
\mathbf{P}\left(U(e)=1 \mid Y\left(V_{n}\right)=\eta^{\prime}\left(V_{n}\right), U\left(E_{n} \backslash\{e\}\right)=\xi^{\prime}\left(E_{n} \backslash\{e\}\right)\right)=\frac{p}{p+(1-p) r_{j}} .
$$

So (10) holds for all $n$ large enough on the event in (i), and (11) holds for all $n$ large enough on the event in (ii). Since the union of these two events has $\mathbf{P}$-probability 1, we have established (9) for $\mathbf{P}$-almost all $(\xi, \eta)$, and the proof is complete.

Remark 6.3. - What we have shown is in fact a statement that is slightly stronger than the one in Theorem 6.2, namely that the required single-edge conditional probabilities for $U\left(E^{Y, j}\right)$ hold even if we condition further on the edge configurations $U\left(E^{Y, j^{\prime}}\right)$ for all $j^{\prime} \neq j$. This observation will in fact be important in Section 9.

\section{7. $k$-Markovianness}

The proof of part (ii) of our main quasilocality result (Theorem 4.4) is sufficiently involved that it is a sensible idea to split it in two halves. In this section, we carry out the first half of the proof, which takes us to a result of independent interest concerning so-called $k$-Markovianness (Theorem 7.2 below).

For a finite vertex set $W \subset \mathbf{Z}^{d}$, define

$$
\partial_{n} W=\{x \in V \backslash W: \exists y \in W \operatorname{such} \text { that } \operatorname{dist}(x, y) \leqslant n\},
$$

where $\operatorname{dist}(\cdot, \cdot)$ denotes graph-theoretic distance. In particular, $\partial_{1} W=\partial W$.

DEFINITION 7.1. - Let $S$ be a finite set, and let $k$ be a positive integer. A probability measure $\mu$ on $S^{\mathbf{Z}^{d}}$ is said to be $k$-Markovian if it admits conditional probabilities such that for all $W \in \mathcal{W}$ all $\xi \in S^{W}$ and all $\eta, \eta^{\prime} \in S^{\mathbf{Z}^{d} \backslash W}$ satisfying $\eta\left(\partial_{k} W\right)=\eta^{\prime}\left(\partial_{k} W\right)$, we have

$$
\mu\left(X(W)=\xi \mid X\left(\mathbf{Z}^{d} \backslash W\right)=\eta\right)=\mu\left(X(W)=\xi \mid X\left(\mathbf{Z}^{d} \backslash W\right)=\eta^{\prime}\right) .
$$

Clearly, for any $k, k$-Markovianness is stronger than quasilocality, since it implies that the modulus in (5) is 0 for all sufficiently large $n$. There are plenty of examples that show that it is in fact strictly stronger; once we have proved Theorem 4.4(i) and Theorem 7.2 below, we see that the fuzzy Potts model with small $\beta$ is such an example.

THEOREM 7.2. - For any $k$ and any $d \geqslant 2$, the fuzzy Potts measure $\mu_{q, \beta,\left(r_{1}, \ldots, r_{s}\right)}^{\mathbf{Z}^{d}, i}$ with $\beta>0$ and the other parameter values as in Theorem 3.2, fails to be $k$-Markovian.

For the proof of this result, we will make use of the following lemma, which is similar to [19, Lemma 5.6]. 
Lemma 7.3. - Fix parameter values $q \in\{3,4, \ldots\}, s \in\{2, \ldots, q-1\},\left(r_{1}, r_{2}, \ldots, r_{s}\right)$, $i \in\{0, \ldots, q\}$ and $\beta>0$ for the fuzzy Potts model on $\mathbf{Z}^{d}$, and let $p=1-\mathrm{e}^{-2 \beta}$. Let $(U, X, Y)$ be as in Theorem 6.2, and write $\mathbf{P}$ for their joint distribution. Fix $x \in \mathbf{Z}^{d}$, let $y_{1}, \ldots, y_{2 d}$ be its nearest neighbors, and for $l=1, \ldots, 2 d$ write $e_{l}$ for the edge $\left\langle x, y_{l}\right\rangle$.

$\mathbf{P}$ admits conditional probabilities such that for all fuzzy spin configurations $\eta \in$ $\{1, \ldots, s\}^{\mathbf{Z}^{d} \backslash\{x\}}$ such that $\eta\left(y_{1}\right)=\eta\left(y_{2}\right)=1$ and $\eta\left(y_{3}\right)=\cdots=\eta\left(y_{2 d}\right)=2$, and all edge configurations $\xi \in\{0,1\}^{E^{d} \backslash\left\{e_{1}, \ldots, e_{2 d}\right\}}$ such that no two of the vertices $y_{3}, \ldots, y_{2 d}$ are connected to each other by a path in $\xi$, we have the following. If $y_{1} \stackrel{\xi}{\leftrightarrow} y_{2}$, then

$$
\begin{aligned}
& \frac{\mathbf{P}\left(Y(x)=1 \mid Y\left(\mathbf{Z}^{d} \backslash\{x\}\right)=\eta, U\left(E^{d} \backslash\left\{e_{1}, \ldots, e_{2 d}\right\}\right)=\xi\right)}{\mathbf{P}\left(Y(x)=2 \mid Y\left(\mathbf{Z}^{d} \backslash\{x\}\right)=\eta, U\left(E^{d} \backslash\left\{e_{1}, \ldots, e_{2 d}\right\}\right)=\xi\right)} \\
& \quad=\left(\frac{p^{2} r_{1}+2 p(1-p) r_{1}+(1-p)^{2} r_{1}^{2}}{(1-p)^{2} r_{1}^{2}}\right)\left(\frac{r_{1}}{r_{2}}\right)\left(\frac{(1-p) r_{2}}{p+(1-p) r_{2}}\right)^{2 d-2},
\end{aligned}
$$

while if $y_{1} \stackrel{\xi}{\leftrightarrow} y_{2}$, then

$$
\begin{aligned}
& \frac{\mathbf{P}\left(Y(x)=1 \mid Y\left(\mathbf{Z}^{d} \backslash\{x\}\right)=\eta, U\left(E^{d} \backslash\left\{e_{1}, \ldots, e_{2 d}\right\}\right)=\xi\right)}{\mathbf{P}\left(Y(x)=2 \mid Y\left(\mathbf{Z}^{d} \backslash\{x\}\right)=\eta, U\left(E^{d} \backslash\left\{e_{1}, \ldots, e_{2 d}\right\}\right)=\xi\right)} \\
& \quad=\left(\frac{p^{2}+2 p(1-p) r_{1}+(1-p)^{2} r_{1}^{2}}{(1-p)^{2} r_{1}^{2}}\right)\left(\frac{r_{1}}{r_{2}}\right)\left(\frac{(1-p) r_{2}}{p+(1-p) r_{2}}\right)^{2 d-2} .
\end{aligned}
$$

A crucial aspect of this result that we will use is that the expression in (12) is strictly greater than the expression in (13); recall that $r_{1}>1$ by the convention (3).

Proof. - By the construction of the fuzzy Potts model from the random-cluster model, we have

$$
\begin{aligned}
& \frac{\mathbf{P}\left(Y(x)=1, U\left(e_{1}\right)=U\left(e_{2}\right)=0 \mid Y\left(\mathbf{Z}^{d} \backslash\{x\}\right)=\eta, U\left(E^{d} \backslash\left\{e_{1}, \ldots, e_{2 d}\right\}\right)=\xi\right)}{\mathbf{P}\left(Y(x)=2, U\left(e_{3}\right)=\cdots=U\left(e_{2 d}\right)=0 \mid Y\left(\mathbf{Z}^{d} \backslash\{x\}\right)=\eta, U\left(E^{d} \backslash\left\{e_{1}, \ldots, e_{2 d}\right)=\xi\right)\right.} \\
& \quad=\frac{\mathbf{P}\left(Y(x)=1 \mid U\left(e_{1}\right)=\cdots=U\left(e_{2 d}\right)=0, Y\left(\mathbf{Z}^{d} \backslash\{x\}\right)=\eta, U\left(E^{d}\left\{e_{1}, \ldots, e_{2 d}\right\}\right)=\xi\right)}{\mathbf{P}\left(Y(x)=2 \mid U\left(e_{1}\right)=\cdots=U\left(e_{2 d}\right)=0, Y\left(\mathbf{Z}^{d} \backslash\{x\}\right)=\eta, U\left(E^{d}\left\{e_{1}, \ldots, e_{2 d}\right\}\right)=\xi\right)} \\
& =\frac{r_{1}}{r_{2}} .
\end{aligned}
$$

By Theorem 6.2 and the defining property (8) of random-cluster measures, we obtain

$$
\begin{aligned}
& \frac{\mathbf{P}\left(Y(x)=2, U\left(e_{3}\right)=\cdots=U\left(e_{2 d}\right)=0 \mid Y\left(\mathbf{Z}^{d} \backslash\{x\}\right)=\eta, U\left(E^{d} \backslash\left\{e_{1}, \ldots, e_{2 d}\right)=\xi\right)\right.}{\mathbf{P}\left(Y(x)=2 \mid Y\left(\mathbf{Z}^{d} \backslash\{x\}\right)=\eta, U\left(E^{d} \backslash\left\{e_{1}, \ldots, e_{2 d}\right)=\xi\right)\right.} \\
& \quad=\left(\frac{(1-p) r_{2}}{p+(1-p) r_{2}}\right)^{2 d-2} .
\end{aligned}
$$

Similarly, we get, on the event $y_{1} \stackrel{\xi}{\leftrightarrow} y_{2}$, that

$$
\begin{aligned}
& \frac{\mathbf{P}\left(Y(x)=1, U\left(e_{1}\right)=U\left(e_{2}\right)=0 \mid Y\left(\mathbf{Z}^{d} \backslash\{x\}\right)=\eta, U\left(E^{d} \backslash\left\{e_{1}, \ldots, e_{2 d}\right\}\right)=\xi\right)}{\mathbf{P}\left(Y(x)=1 \mid Y\left(\mathbf{Z}^{d} \backslash\{x\}\right)=\eta, U\left(E^{d} \backslash\left\{e_{1}, \ldots, e_{2 d}\right)=\xi\right)\right.} \\
& =\left(\frac{(1-p) r_{1}}{p+(1-p) r_{1}}\right)^{2}=\frac{(1-p)^{2} r_{1}^{2}}{p^{2}+2 p(1-p) r_{1}+(1-p)^{2} r_{1}^{2}},
\end{aligned}
$$


while on the event $y_{1} \stackrel{\xi}{\leftrightarrow} y_{2}$ we get

$$
\begin{aligned}
& \frac{\mathbf{P}\left(Y(x)=1, U\left(e_{1}\right)=U\left(e_{2}\right)=0 \mid Y\left(\mathbf{Z}^{d} \backslash\{x\}\right)=\eta, U\left(E^{d} \backslash\left\{e_{1}, \ldots, e_{2 d}\right\}\right)=\xi\right)}{\mathbf{P}\left(Y(x)=1 \mid Y\left(\mathbf{Z}^{d} \backslash\{x\}\right)=\eta, U\left(E^{d} \backslash\left\{e_{1}, \ldots, e_{2 d}\right)=\xi\right)\right.} \\
& =\frac{(1-p)^{2} r_{1}^{2}}{p^{2} r_{1}+2 p(1-p) r_{1}+(1-p)^{2} r_{1}^{2}} .
\end{aligned}
$$

Combining (14), (15) and (17), we get, still on the event $y_{1} \stackrel{\xi}{\leftrightarrow} y_{2}$,

$$
\begin{aligned}
& \frac{\mathbf{P}\left(Y(x)=1 \mid Y\left(\mathbf{Z}^{d} \backslash\{x\}\right)=\eta, U\left(E^{d} \backslash\left\{e_{1}, \ldots, e_{2 d}\right)=\xi\right)\right.}{\mathbf{P}\left(Y(x)=2 \mid Y\left(\mathbf{Z}^{d} \backslash\{x\}\right)=\eta, U\left(E^{d} \backslash\left\{e_{1}, \ldots, e_{2 d}\right)=\xi\right)\right.} \\
& =\frac{\mathbf{P}\left(Y(x)=1 \mid Y\left(\mathbf{Z}^{d} \backslash\{x\}\right)=\eta, U\left(E^{d} \backslash\left\{e_{1}, \ldots, e_{2 d}\right\}\right)=\xi\right)}{\mathbf{P}\left(Y(x)=1, U\left(e_{1}\right)=U\left(e_{2}\right)=0 \mid Y\left(\mathbf{Z}^{d} \backslash\{x\}\right)=\eta, U\left(E^{d} \backslash\left\{e_{1}, \ldots, e_{2 d}\right\}\right)=\xi\right)} \\
& \times \frac{\mathbf{P}\left(Y(x)=1, U\left(e_{1}\right)=U\left(e_{2}\right)=0 \mid Y\left(\mathbf{Z}^{d} \backslash\{x\}\right)=\eta, U\left(E^{d} \backslash\left\{e_{1}, \ldots, e_{2 d}\right\}\right)=\xi\right)}{\mathbf{P}\left(Y(x)=2, U\left(e_{3}\right)=\cdots=U\left(e_{2 d}\right)=0 \mid Y\left(\mathbf{Z}^{d} \backslash\{x\}\right)=\eta, U\left(E^{d} \backslash\left\{e_{1}, \ldots, e_{2 d}\right\}\right)=\xi\right)} \\
& \times \frac{\mathbf{P}\left(Y(x)=2, U\left(e_{3}\right)=\cdots=U\left(e_{2 d}\right)=0 \mid Y\left(\mathbf{Z}^{d} \backslash\{x\}\right)=\eta, U\left(E^{d} \backslash\left\{e_{1}, \ldots, e_{2 d}\right\}\right)=\xi\right)}{\mathbf{P}\left(Y(x)=2 \mid Y\left(\mathbf{Z}^{d} \backslash\{x\}\right)=\eta, U\left(E^{d} \backslash\left\{e_{1}, \ldots, e_{2 d}\right)=\xi\right)\right.} \\
& =\left(\frac{p^{2} r_{1}+2 p(1-p) r_{1}+(1-p)^{2} r_{1}^{2}}{(1-p)^{2} r_{1}^{2}}\right)\left(\frac{r_{1}}{r_{2}}\right)\left(\frac{(1-p) r_{2}}{p+(1-p) r_{2}}\right)^{2 d-2}
\end{aligned}
$$

and (12) is established. A similar calculation for $y_{1} \stackrel{\xi}{\leftrightarrow} y_{2}$ yields (13) from (14), (15) and (16), completing the proof.

For the proofs of Theorems 4.4(ii) and 7.2, it is useful to find a fuzzy spin configuration $\eta^{*}$ that can serve as a "point of discontinuity" in (5). To this end, define $\eta^{*} \in\{1, \ldots, s\}^{\mathbf{Z}^{d} \backslash\{\boldsymbol{0}\}}$, where $\mathbf{0}$ is the origin, as follows. For each $x=\left(x_{1}, \ldots, x_{d}\right) \in$ $\mathbf{Z}^{d} \backslash\{\mathbf{0}\}$, set

$$
\eta^{*}(x)= \begin{cases}2 & \text { if } x_{1}=0 \text { and }\left|x_{2}\right|+\left|x_{3}\right|+\cdots+\left|x_{d}\right|=1, \\ \text { or if } x_{1}=-1 \text { and }\left|x_{2}\right|+\left|x_{3}\right|+\cdots+\left|x_{d}\right|=1, \\ 1 & \text { otherwise }\end{cases}
$$

(This is essentially the same configuration as the one defined in [19] for the same purposes in the so-called divide-and-color model. That configuration was in turn inspired by one used in [9], again for the purpose of proving nonquasilocality; see also [21] and [22] for other applications of the same circle of ideas.) See Fig. 1 for an illustration of $\eta^{*}$ in $d=2$ dimensions. Each of the $2 d$ nearest neighbors of $\mathbf{0}$ are in different connected fuzzy spin components of $\eta^{*}$ : two of them are infinite components of 1's, each filling up essentially a half-space, while the remaining $2 d-2$ of them are singleton components of 2's.

Proof of Theorem 7.2. - Let $(U, X, Y)$ be defined as before, and write $\mathbf{P}$ for their joint distribution. As in Section 6, let $\Lambda_{n}=\{-n, \ldots, n\}^{d}$. For each $k$, define the fuzzy spin 


$\begin{array}{lllllllllllllll}1 & 1 & 1 & 1 & 1 & 1 & 2 & 1 & 1 & 1 & 1 & 1 & 1 & 1 & 1 \\ 1 & 1 & 1 & 1 & 1 & 1 & 2 & 1 & 1 & 1 & 1 & 1 & 1 & 1 & 1 \\ 1 & 1 & 1 & 1 & 1 & 1 & 2 & 1 & 1 & 1 & 1 & 1 & 1 & 1 & 1 \\ 1 & 1 & 1 & 1 & 1 & 1 & 2 & 1 & 1 & 1 & 1 & 1 & 1 & 1 & 1 \\ 1 & 1 & 1 & 1 & 1 & 1 & 2 & 1 & 1 & 1 & 1 & 1 & 1 & 1 & 1 \\ 1 & 1 & 1 & 1 & 1 & 1 & 2 & 1 & 1 & 1 & 1 & 1 & 1 & 1 & 1 \\ 1 & 1 & 1 & 1 & 1 & 1 & 1 & 2 & 1 & 1 & 1 & 1 & 1 & 1 & 1 \\ 1 & 1 & 1 & 1 & 1 & 1 & 1 & x & 1 & 1 & 1 & 1 & 1 & 1 & 1 \\ 1 & 1 & 1 & 1 & 1 & 1 & 1 & 2 & 1 & 1 & 1 & 1 & 1 & 1 & 1 \\ 1 & 1 & 1 & 1 & 1 & 1 & 2 & 1 & 1 & 1 & 1 & 1 & 1 & 1 & 1 \\ 1 & 1 & 1 & 1 & 1 & 1 & 2 & 1 & 1 & 1 & 1 & 1 & 1 & 1 & 1 \\ 1 & 1 & 1 & 1 & 1 & 1 & 2 & 1 & 1 & 1 & 1 & 1 & 1 & 1 & 1 \\ 1 & 1 & 1 & 1 & 1 & 1 & 2 & 1 & 1 & 1 & 1 & 1 & 1 & 1 & 1 \\ 1 & 1 & 1 & 1 & 1 & 1 & 2 & 1 & 1 & 1 & 1 & 1 & 1 & 1 & 1 \\ 1 & 1 & 1 & 1 & 1 & 1 & 2 & 1 & 1 & 1 & 1 & 1 & 1 & 1 & 1\end{array}$

Fig. 1. The fuzzy spin configuration $\eta^{*}$ restricted to the box $\Lambda_{7}=\{-7, \ldots, 7\}^{2}$ in two dimensions. The $x$ in the middle is the origin, whose value is not determined by $\eta^{*}$.

configurations $\eta^{1, k}, \eta^{2, k} \in\{1, \ldots, s\}^{\Lambda_{k+1} \backslash\{0\}}$ by setting

$$
\eta^{1, k}(x)= \begin{cases}1 & \text { for } x \in \Lambda_{k+1} \backslash \Lambda_{k}, \\ \eta^{*}(x) & \text { for } x \in \Lambda_{k} \backslash\{\mathbf{0}\}\end{cases}
$$

and

$$
\eta^{2, k}(x)= \begin{cases}2 & \text { for } x \in \Lambda_{k+1} \backslash \Lambda_{k}, \\ \eta^{*}(x) & \text { for } x \in \Lambda_{k} \backslash\{\mathbf{0}\} .\end{cases}
$$

Note that $\eta^{1, k}=\eta^{2, k}$ on $\Lambda_{k} \backslash\{\boldsymbol{0}\}$. Hence, in order to show that $Y$ is not $k$-Markovian, it is enough to show that

$$
\mathbf{P}\left(Y(\mathbf{0})=j \mid Y\left(\Lambda_{k+1} \backslash\{\mathbf{0}\}\right)=\eta^{1, k}\right) \neq \mathbf{P}\left(Y(\mathbf{0})=j \mid Y\left(\Lambda_{k+1} \backslash\{\mathbf{0}\}\right)=\eta^{2, k}\right)
$$

for some $j \in\{1, \ldots, s\}$. We may assume that

$$
\mathbf{P}\left(Y(\mathbf{0})=2 \mid Y\left(\Lambda_{k+1} \backslash\{\mathbf{0}\}\right)=\eta^{1, k}\right)=\mathbf{P}\left(Y(\mathbf{0})=2 \mid Y\left(\Lambda_{k+1} \backslash\{\mathbf{0}\}\right)=\eta^{2, k}\right)
$$

because if this fails, then we are done. ${ }^{2}$ Write $b$ for the conditional probability in (20), write $c_{1}$ and $c_{2}$ for the expressions in (12) and (13) respectively, and recall that $c_{1}>c_{2}$. We furthermore have $b>0$ due to Lemma 4.5. Write $y_{1}$ and $y_{2}$ for the two nearest neighbors of $\mathbf{0}$ that take fuzzy spin value 1 in $\eta^{*}$, and let $A$ denote the event that $U$ contains a path from $y_{1}$ to $y_{2}$ that does not pass through $\mathbf{0}$. Observe that $Y\left(\Lambda_{k+1} \backslash\{\mathbf{0}\}\right)=\eta^{2, k}$ implies $\neg A$, so that by Lemma 7.3 we get

$$
\mathbf{P}\left(Y(\mathbf{0})=1 \mid Y\left(\Lambda_{k+1} \backslash\{\mathbf{0}\}\right)=\eta^{2, k}\right)=c_{2} b .
$$

On the other hand, we clearly have

$$
\mathbf{P}\left(A \mid Y\left(\Lambda_{k+1} \backslash\{\mathbf{0}\}\right)=\eta^{1, k}\right)>0
$$

\footnotetext{
${ }^{2}$ In fact it does fail. Our argument nevertheless seems like the most streamlined way to prove the result.
} 
so that, by another application of Lemma 7.3, we have

$$
\begin{aligned}
\mathbf{P} & \left.Y(0)=1 \mid Y\left(\Lambda_{k+1} \backslash\{\mathbf{0}\}\right)=\eta^{1, k}\right) \\
& =c_{1} b \mathbf{P}\left(A \mid Y\left(\Lambda_{k+1} \backslash\{\mathbf{0}\}\right)=\eta^{1, k}\right)+c_{2} b \mathbf{P}\left(\neg A \mid y\left(\Lambda_{k+1} \backslash\{\mathbf{0}\}\right)=\eta^{1, k}\right) \\
& =c_{2} b+\left(c_{1}-c_{2}\right) b \mathbf{P}\left(A \mid Y\left(\Lambda_{k+1} \backslash\{\mathbf{0}\}\right)=\eta^{1, k}\right) \\
& >c_{2} b \\
& =\mathbf{P}\left(Y(\mathbf{0})=1 \mid Y\left(\Lambda_{k+1} \backslash\{\mathbf{0}\}\right)=\eta^{2, k}\right) .
\end{aligned}
$$

Hence, (19) is established for $j=1$, and the proof is complete.

\section{Proof of the main result for large $\beta$}

In this section, we will prove non-quasilocality of the fuzzy Potts model for large $\beta$. More precisely, we will prove Theorem 4.4(ii), thereby establishing also Theorem 3.2(ii) concerning non-Gibbsianness at large $\beta$.

To this end, we will sharpen the proof of Theorem 7.2. The thing we need to do compared to that proof, is to demonstrate that the discrepancy between the left-hand side and the right-hand side of (19) not only is nonzero for all $k$, but that it is in fact bounded away from 0 uniformly in $k$. The way to do this is to show that the conditional probability in (21) is bounded away from 0 uniformly in $k$. This can be done for the range of $\beta$-values given in Theorem 4.4(ii), but the argument simplifies somewhat if we instead consider the following modifications of the fuzzy spin configurations $\eta^{1, k}$ and $\eta^{2, k}$ considered in the proof of Theorem 7.2. Define, for positive integers $k$ and $n$ such that $k<n$, the configurations $\eta^{1, k, n}, \eta^{2, k, n} \in\{1, \ldots, s\}^{\Lambda_{n} \backslash\{0\}}$ as

$$
\eta^{1, k, n}(x)= \begin{cases}1 & \text { for } x \in \Lambda_{n} \backslash \Lambda_{k}, \\ \eta^{*}(x) & \text { for } x \in \Lambda_{k} \backslash\{\mathbf{0}\}\end{cases}
$$

and

$$
\eta^{2, k, n}(x)= \begin{cases}2 & \text { for } x \in \Lambda_{n} \backslash \Lambda_{k}, \\ \eta^{*}(x) & \text { for } x \in \Lambda_{k} \backslash\{\mathbf{0}\},\end{cases}
$$

where $\eta^{*} \in\{1, \ldots, s\}^{\mathbf{Z}^{d} \backslash\{0\}}$ is defined as in (18). Specifically, we will prove the following result, which will easily imply Theorem 4.4(ii).

Lemma 8.1.-Suppose that we pick $Y$ according to the fuzzy Potts measure $\mu_{q, \beta,\left(r_{1}, \ldots, r_{s}\right)}^{\mathbf{Z}^{d}, i}$ with $d \geqslant 2, \beta>\frac{1}{2} \log \left(\left(1+\left(r_{1}-1\right) p_{c}(d)\right) /\left(1-p_{c}(d)\right)\right)$, and the other parameter values as in Theorem 3.2. Then there exists a constant $\alpha>0$ (depending on $d, r_{1}$ and $\beta$, but not on $k$ ) such that for all $k$ and all sufficiently large $n$ (depending on $k$ ), we have

$$
\begin{aligned}
& \mathbf{P}\left(Y(\mathbf{0})=1 \mid Y(\mathbf{0}) \in\{1,2\}, Y\left(\Lambda_{n} \backslash\{\mathbf{0}\}\right)=\eta^{1, k, n}\right) \\
& \quad-\mathbf{P}\left(Y(\mathbf{0})=1 \mid Y(\mathbf{0}) \in\{1,2\}, Y\left(\Lambda_{n} \backslash\{\mathbf{0}\}\right)=\eta^{2, k, n}\right) \geqslant \alpha .
\end{aligned}
$$

The proof of this lemma is based on comparing the random-cluster configuration $U$ to supercritical i.i.d. percolation. The key result for such percolation processes that we 
need, is captured in the next lemma. Recall that $p_{c}(d)$ is the critical value for i.i.d. bond percolation on $\mathbf{Z}^{d}$. Let $y_{1}, y_{2} \in \mathbf{Z}^{d}$ be as in the proof of Theorem 7.2; to be more specific we take $y_{1}$ to be the nearest neighbor of $\mathbf{0}$ whose first coordinate is -1 , and $y_{2}$ to be the nearest neighbor of $\mathbf{0}$ whose first coordinate is +1 . Also as in that proof, define

$A=\left\{\exists\right.$ a path of open edges from $y_{1}$ to $y_{2}$ that does not pass through $\left.\mathbf{0}\right\}$.

LEMMA 8.2. - For any $k$, let $E^{d, k}$ denote the set of edges in $E^{d}$ that are incident either to $\mathbf{0}$ or to some $x \in \Lambda_{k} \backslash\{\mathbf{0}\}$ with $\eta^{*}(x)=2$. For $\tilde{p} \in[0,1]$, consider the inhomogeneous bond percolation process $\widetilde{U} \in\{0,1\}^{E^{d}}$ obtained by setting $\widetilde{U}(e)=0$ for all $e \in E^{d, k}$, and for each $e \in E^{d} \backslash E^{d, k}$ independently setting

$$
\widetilde{U}(e)= \begin{cases}1 & \text { with probability } \tilde{p}, \\ 0 & \text { with probability } 1-\tilde{p} .\end{cases}
$$

Write $P_{\tilde{p}, k}$ for the corresponding probability measure on $\{0,1\}^{E^{d}}$. If $\tilde{p}>p_{c}(d)$, then

$$
\lim _{k \rightarrow \infty} P_{\tilde{p}, k}(A)>0
$$

and the limit is decreasing.

Proof. - Note first that $P_{\tilde{p}, k}(A)$ is decreasing in $k$, since the measures $P_{\tilde{p}, k}$ are stochastically decreasing in $k$ and $A$ is an increasing event. Hence the limit in (23) exists, and it only remains to show that $P_{\tilde{p}, k}(A)$ is bounded away from 0 uniformly in $k$. Define the events $A_{1}, A_{2}, A_{3} \in\{0,1\}^{E^{d}}$ as follows.

$A_{1}=\left\{\exists\right.$ an infinite open path starting at $y_{1}$ and contained in the left half-space $\}$,

$A_{2}=\left\{\exists\right.$ an infinite open path starting at $y_{2}$ and contained in the right half-space $\}$,

$A_{3}=\{\exists$ a unique infinite open cluster $\}$.

By the left (resp. right) half-space, we here mean the set of vertices whose first coordinate is strictly negative (resp. strictly positive), together with edges connecting such vertices. Note that

$$
A_{1} \cap A_{2} \cap A_{3} \subseteq A .
$$

Barsky, Grimmett and Newman [3] showed that for i.i.d. bond percolation in a half-space with $p>p_{c}(d)$, there a.s. exists an infinite open cluster, and hence that any given vertex has strictly positive probability of being in an infinite cluster. This immediately implies that $P_{\tilde{p}, k}\left(A_{1}\right)$ and $P_{\tilde{p}, k}\left(A_{2}\right)$ both are bounded away from 0 when $\tilde{p}>p_{c}(d)$. Note also that $A_{1}$ and $A_{2}$ are independent under $P_{\tilde{p}, k}$, so that $P_{\tilde{p}, k}\left(A_{1} \cap A_{2}\right)=P_{\tilde{p}, k}\left(A_{1}\right) P_{\tilde{p}, k}\left(A_{2}\right)$ is also bounded away from 0 .

Next, recall the famous a.s. uniqueness of the infinite cluster result for i.i.d. bond percolation on $\mathbf{Z}^{d}$, established by Aizenman, Kesten and Newman [2]. But $P_{\tilde{p}, k}$ arises by conditioning such a percolation process on an event of positive probability, namely the event that all edges in $E^{d, k}$ are closed. Hence any a.s. result for i.i.d. percolation carries over to $P_{\tilde{p}, k}$, so that in particular $P_{\tilde{p}, k}\left(A_{3}\right)=1$. We get 


$$
\begin{aligned}
\lim _{k \rightarrow \infty} P_{\tilde{p}, k}(A) & \geqslant \lim _{k \rightarrow \infty} P_{\tilde{p}, k}\left(A_{1} \cap A_{2} \cap A_{3}\right)=\lim _{k \rightarrow \infty} P_{\tilde{p}, k}\left(A_{1} \cap A_{2}\right) \\
& =\lim _{k \rightarrow \infty} P_{\tilde{p}, k}\left(A_{1}\right) P_{\tilde{p}, k}\left(A_{2}\right)>0
\end{aligned}
$$

as desired.

For the proof of Lemma 8.1, we need also to recall the concept of stochastic domination. Let $E$ be a finite or countable set (in our applications, $E$ will be an edge set; hence the notation). For $\xi, \xi^{\prime} \in\{0,1\}^{E}$, write $\xi \preccurlyeq \xi^{\prime}$ if $\xi(e) \leqslant \xi^{\prime}(e)$ for all $e \in E$. A function $f:\{0,1\}^{E} \rightarrow \mathbf{R}$ is said to be increasing if $f(\xi) \leqslant f\left(\xi^{\prime}\right)$ whenever $\xi \leqslant \xi^{\prime}$. For two probability measures $\phi$ and $\phi^{\prime}$ on $\{0,1\}^{E}$, we say that $\phi$ is stochastically dominated by $\phi^{\prime}$, writing $\phi \stackrel{\mathcal{D}}{\preccurlyeq} \phi^{\prime}$, if

$$
\int_{\{0,1\}^{E}} f d \phi \leqslant \int_{\{0,1\}^{E}} f d \phi^{\prime}
$$

holds for all bounded increasing $f:\{0,1\}^{E} \rightarrow \mathbf{R}$. It is well known (Strassen's Theorem) that $\phi \stackrel{\mathcal{D}}{\preccurlyeq} \phi^{\prime}$ is equivalent to the existence of a coupling of two $\{0,1\}^{E}$-valued random objects $U$ and $U^{\prime}$, with respective distributions $\phi$ and $\phi^{\prime}$, such that $U \preccurlyeq U^{\prime}$ with probability 1.

Proof of Lemma 8.1. - Define $A_{n} \in\{0,1\}^{E^{d}}$ to be the event that there exists a path of open edges from $y_{1}$ to $y_{2}$ that does not pass through $\{\mathbf{0}\}$ and that is contained in $\Lambda_{n}$. Note that $A_{1}, A_{2}, \ldots$ is an increasing sequence of events converging to $A$, so that

$$
\lim _{n \rightarrow \infty} P_{\tilde{p}, k}\left(A_{n}\right)=P_{\tilde{p}, k}(A)
$$

for any $\tilde{p}$ and $k$.

Write $\gamma=\gamma(\tilde{p})$ for the limit in (23). Due to (24), we can find, for each $k$, an $n=n(k, \tilde{p})$ such that

$$
P_{\tilde{p}, k}\left(A_{n}\right) \geqslant \frac{\gamma}{2}
$$

Set $p=1-\mathrm{e}^{-2 \beta}$ and $\tilde{p}=p /\left(p+(1-p) r_{1}\right)$, and note that due to our assumption on $\beta$ we have $\tilde{p}>p_{c}(d)$. Recall Theorem 6.2 , and consider the conditional distribution of $U\left(E^{Y, 1}\right)$ given $Y$. If we reveal the status of the edges in $E^{Y, 1}$ one at a time, then at each stage we have conditional probability (given $Y$ and all the edges seen so far) at least $\tilde{p}$ of seeing an open edge; this is due to the defining property (6.1) of random-cluster measures. It follows that the conditional distribution of $U\left(E^{Y, 1}\right)$ given $Y$ stochastically dominates the projection of $P_{\tilde{p}, k}$ on $\{0,1\}^{E^{Y, 1}}$. If we now pick $n$ large enough so that (25) holds, then (due to the fact that $A_{n}$ is an increasing event) we get

$$
\begin{aligned}
& \mathbf{P}\left(A \mid Y(\mathbf{0}) \in\{1,2\}, Y\left(\Lambda_{n} \backslash\{\mathbf{0}\}\right)=\eta^{1, k, n}\right) \\
& \quad \geqslant \mathbf{P}\left(A_{n} \mid Y(\mathbf{0}) \in\{1,2\}, Y\left(\Lambda_{n} \backslash\{\mathbf{0}\}\right)=\eta^{1, k, n}\right) \geqslant P_{\tilde{p}, k}\left(A_{n}\right) \geqslant \frac{\gamma}{2} .
\end{aligned}
$$

Write (as in the proof of Theorem 7.2) $c_{1}$ and $c_{2}$ for the right-hand sides of (12) and (13), and recall again that $c_{1}>c_{2}$. Lemma 7.3 implies 


$$
\begin{aligned}
\mathbf{P}(Y( & \left.\{0\})=1 \mid Y(\mathbf{0}) \in\{1,2\}, Y\left(\Lambda_{n} \backslash\{\mathbf{0}\}\right)=\eta^{1, k, n}\right) \\
= & \left(\mathbf{P}\left(Y(\{0\})=1 \mid Y(\mathbf{0}) \in\{1,2\}, Y\left(\Lambda_{n} \backslash\{\mathbf{0}\}\right)=\eta^{1, k, n}, A\right)\right. \\
& \left.\times \mathbf{P}\left(A \mid Y(\mathbf{0}) \in\{1,2\}, Y\left(\Lambda_{n} \backslash\{\mathbf{0}\}\right)=\eta^{1, k, n}\right)\right) \\
& +\left(\mathbf{P}\left(Y(\{0\})=1 \mid Y(\mathbf{0}) \in\{1,2\}, Y\left(\Lambda_{n} \backslash\{\mathbf{0}\}\right)=\eta^{1, k, n}, \neg A\right)\right. \\
& \left.\times \mathbf{P}\left(\neg A \mid Y(\mathbf{0}) \in\{1,2\}, Y\left(\Lambda_{n} \backslash\{\mathbf{0}\}\right)=\eta^{1, k, n}\right)\right) \\
= & \frac{c_{1}}{c_{1}+1} \mathbf{P}\left(A \mid Y(\mathbf{0}) \in\{1,2\}, Y\left(\Lambda_{n} \backslash\{\mathbf{0}\}\right)=\eta^{1, k, n}\right) \\
& +\frac{c_{2}}{c_{2}+1} \mathbf{P}\left(\neg A \mid Y(\mathbf{0}) \in\{1,2\}, Y\left(\Lambda_{n} \backslash\{\mathbf{0}\}\right)=\eta^{1, k, n}\right) \\
= & \frac{c_{2}}{c_{2}+1}+\left(\frac{c_{1}}{c_{1}+1}-\frac{c_{2}}{c_{2}+1}\right) \mathbf{P}\left(A \mid Y(\mathbf{0}) \in\{1,2\}, Y\left(\Lambda_{n} \backslash\{\mathbf{0}\}\right)=\eta^{1, k, n}\right) \\
\geqslant & \frac{c_{2}}{c_{2}+1}+\frac{\gamma}{2}\left(\frac{c_{1}}{c_{1}+1}-\frac{c_{2}}{c_{2}+1}\right) .
\end{aligned}
$$

On the other hand,

$$
\mathbf{P}\left(Y(\{0\})=1 \mid Y(\mathbf{0}) \in\{1,2\}, Y\left(\Lambda_{n} \backslash\{\mathbf{0}\}\right)=\eta^{2, k, n}\right)=\frac{c_{2}}{c_{2}+1},
$$

again by Lemma 7.3. Lemma 8.1 therefore follows with $\alpha=(\gamma / 2)\left(c_{1} /\left(c_{1}+1\right)-\right.$ $\left.c_{2} /\left(c_{2}+1\right)\right)$.

Proof of Theorem 4.4(ii). - For each $k$, choose an $n=n(k)$ such that (22) holds. Assume for contradiction that $\mu_{q, \beta,\left(r_{1}, \ldots, r_{s}\right)}^{\mathbf{Z}^{d}, i}$ is quasilocal. Then the limits

$$
\lim _{k \rightarrow \infty} \mathbf{P}\left(Y(\mathbf{0}) \in\{1,2\} \mid Y\left(\Lambda_{n} \backslash\{\mathbf{0}\}\right)=\eta^{1, k, n}\right)
$$

and

$$
\lim _{k \rightarrow \infty} \mathbf{P}\left(Y(\mathbf{0}) \in\{1,2\} \mid Y\left(\Lambda_{n} \backslash\{\mathbf{0}\}\right)=\eta^{2, k, n}\right)
$$

must exist and be equal. By Lemma 4.5, this common limit must be nonzero; let us denote it by $\delta$. Lemma 8.1 then yields

$$
\liminf _{k \rightarrow \infty}\left(\mathbf{P}\left(Y(\mathbf{0})=1 \mid Y\left(\Lambda_{n} \backslash\{\mathbf{0}\}\right)=\eta^{1, k, n}\right)-\mathbf{P}\left(Y(\mathbf{0})=1 \mid Y\left(\Lambda_{n} \backslash\{\mathbf{0}\}\right)=\eta^{2, k, n}\right)\right) \geqslant \alpha \delta,
$$

violating the quasilocality condition (5).

Let us end this section by saying a few words about Conjecture 3.3. The comparison we made in the proof of Lemma 4.5 between random-cluster measures and i.i.d. percolation appears to be rather crude, and is likely to be improvable in such a way that the range of $\beta$-values for which the conclusion holds is enlarged. It even seems likely that if $n=n(k)$ grows sufficiently fast, then

the conditional probabilities of $A$ in (26) should be bounded away from 0 as soon as $\phi_{p, r_{1}}^{\mathbf{Z}^{d}}$,free creates an infinite cluster; that is, as soon as $\beta>\beta_{c}\left(d, r_{1}\right)$. 
If this could be established, then we would have a stronger version of Lemma 8.2, that would allow us to extend the conclusions of Lemma 8.1 and Theorems 4.4(ii) and 3.2(ii) to all $\beta>\beta_{c}\left(d, r_{1}\right)$, thereby establishing Conjecture 3.3.

It would be possible to prove (27) by modifying our arguments in the proof of Lemma 8.2, provided that we had a random-cluster analogue of the result in [3] on existence of infinite clusters in half-spaces for supercritical percolation. To prove such a result for the random-cluster model is still an open problem; a positive answer would follow from (and is in fact equivalent to) a conjecture by Pisztora [27, bottom of p. 441].

\section{Proof of the main result for small $\beta$}

In this final section, we will prove Theorem 4.4(i), which implies Theorem 3.2(i). We begin with an easy result about the random-cluster model.

LEMma 9.1. - Let $G=(V, E)$ be a finite graph, and let $\left(V_{1}, V_{2}\right)$ be an arbitrary bipartition of $V$. Define

$$
\begin{aligned}
& E_{1}=\left\{e \in E: \text { both endpoints of } e \text { are in } V_{1}\right\}, \\
& E_{2}=\left\{e \in E: \text { both endpoints of } e \text { are in } V_{2}\right\}, \\
& E_{3}=\left\{e \in E: \text { one endpoint of } e \text { is in } V_{1} \text { and the other in } V_{2}\right\} .
\end{aligned}
$$

Define also $G_{1}=\left(V_{1}, E_{1}\right)$ and $G_{2}=\left(V_{2}, E_{2}\right)$. Fix $p \in[0,1]$ and $q>0$, and suppose that we pick the random edge configuration $U \in\{0,1\}^{E}$ according to the random-cluster measure $\phi_{p, q}^{G}$ conditional on the event $A_{E_{3}} \subset\{0,1\}^{E}$ defined as

$$
A_{E_{3}}=\left\{\xi \in\{0,1\}^{E}: \xi(e)=0 \text { for all } e \in E_{3}\right\} .
$$

Then $U\left(E_{1}\right)$ and $U\left(E_{2}\right)$ are independent of each other, with respective distributions $\phi_{p, q}^{G_{1}}$ and $\phi_{p, q}^{G_{2}}$.

Proof. - Write $P$ for the distribution of $U$. For $\xi \in A_{E_{3}}$, write $k(\xi)$ for the number of connected components of $\xi$, and write $k_{1}(\xi)$ and $k_{2}(\xi)$ for the number of connected components of $\xi$ contained in $G_{1}$ and in $G_{2}$, respectively; note that $\xi=\xi_{1}+\xi_{2}$. The definition (7) of random-cluster measures yields

$$
\begin{aligned}
P(\xi) & =\frac{\phi_{p, q}^{G}(\xi)}{\phi_{p, q}^{G}\left(A_{E_{3}}\right)}=\frac{q^{k(\xi)}}{\widehat{Z}_{p, q}^{G} \phi_{p, q}^{G}\left(A_{E_{3}}\right)} \prod_{e \in E} p^{\xi(e)}(1-p)^{(1-\xi(e))} \\
& =\left(\frac{q^{k_{1}(\xi)}}{\widehat{Z}_{p, q}^{G_{1}}} \prod_{e \in E_{1}} p^{\xi(e)}(1-p)^{(1-\xi(e))}\right)\left(\frac{q^{k_{2}(\xi)}}{\widehat{Z}_{p, q}^{G_{2}}} \prod_{e \in E_{2}} p^{\xi(e)}(1-p)^{(1-\xi(e))}\right) \\
& =\phi_{p, q}^{G_{1}}\left(\xi\left(E_{1}\right)\right) \phi_{p, q}^{G_{2}}\left(\xi\left(E_{2}\right)\right)
\end{aligned}
$$

because $\widehat{Z}_{p, q}^{G_{1}} \widehat{Z}_{p, q}^{G_{2}}$ must equal $\widehat{Z}_{p, q}^{G} \phi_{p, q}^{G}\left(A_{E_{3}}\right)$ by normalization.

Recall our earlier notation that $\mathbf{P}$ is the joint distribution of $(U, X, Y)$. Now we need to work with such distributions for more than one graph simultaneously, so we write $\mathbf{P}^{G}$ to emphasize on which graphs $(U, X, Y)$ lives. 
Let $G, G_{1}, G_{2}, E_{3}$ and $A_{E_{3}}$ be as in Lemma 9.1. Imagine picking $(U, X, Y)$ according to $\mathbf{P}^{G}$, and write $\mathbf{P}^{G} \mid A_{E_{3}}$ f or the conditional distribution of $(U, X, Y)$ given the event $A_{E_{3}}$. It follows from Lemma 9.1 and the construction of $X$ and $Y$ from $U$ that an edge-spin triple $(U, X, Y)$ with distribution $\mathbf{P}^{G} \mid A_{E_{3}}$ may alternatively be obtained by

(i) setting $U\left(E_{3}\right) \equiv 0$,

(ii) picking $\left(U\left(E_{1}\right), X\left(V_{1}\right), Y\left(V_{1}\right)\right)$ according to $\mathbf{P}^{G_{1}}$, and

(iii) picking $\left(U\left(E_{2}\right), X\left(V_{2}\right), Y\left(V_{2}\right)\right)$ according to $\mathbf{P}^{G_{2}}$, where (ii) and (iii) are done independently. An analogous result on $\mathbf{Z}^{d}$ is the following.

Lemma 9.2. - Let $V \subset \mathbf{Z}^{d}$ be a finite subset of $\mathbf{Z}^{d}$, and define

$$
\begin{aligned}
& E_{1}=\left\{e \in E^{d}: \text { both endpoints of } e \text { are in } V\right\}, \\
& E_{2}=\left\{e \in E^{d}: \text { both endpoints of } e \text { are in } \mathbf{Z}^{d} \backslash V\right\}, \\
& E_{3}=\left\{e \in E^{d}: \text { exactly one endpoint of } e \text { is in } V\right\}
\end{aligned}
$$

and $G=\left(V, E_{1}\right)$. Pick $(U, X, Y) \in\{0,1\}^{E^{d}} \times\{1, \ldots, q\}^{\mathbf{Z}^{d}} \times\{1, \ldots, s\}^{\mathbf{Z}^{d}}$ as in Theorem 6.2, and write $\mathbf{P}^{\mathbf{Z}^{d}}$ for their joint distribution. Then the $\mathbf{P}^{\mathbf{Z}^{d}}$-conditional distribution given $A_{E_{3}}$ and any event defined in terms of $\left(U\left(E_{1}\right), X\left(\mathbf{Z}^{d} \backslash V\right), Y\left(\mathbf{Z}^{d} \backslash V\right)\right)$, is $\mathbf{P}^{G}$.

Proof. - Pick $n$ large enough so that $V \subset \Lambda_{n}$. By the reasoning prior to the lemma,

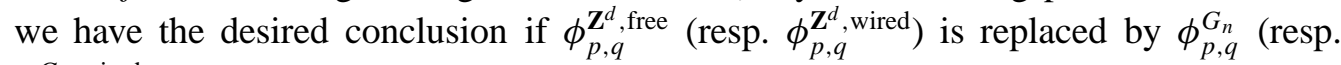
$\left.\phi_{p, q}^{G_{n}, \text { wired }}\right)$ in the construction of $(U, X, Y)$. The lemma now follows by taking limits as $n \rightarrow \infty$.

Let us now give a rough outline of the proof of Theorem 4.4(i). Fix an arbitrary finite vertex set $W \subset \mathbf{Z}^{d}$, together with a fuzzy spin configuration $\xi \in\{1, \ldots, s\}^{W}$. To show that the fuzzy Potts measure $\mu_{q, \beta,\left(r_{1}, \ldots, r_{s}\right)}^{\mathbf{Z}^{d}, i}$ is quasilocal, we need to show for any $\varepsilon>0$ that there exists an $n<\infty$ such that

$$
\begin{aligned}
& \mid \mu_{q, \beta,\left(r_{1}, \ldots, r_{s}\right)}^{\mathbf{Z}^{d}, i}\left(Y(W)=\xi \mid Y\left(\mathbf{Z}^{d} \backslash W\right)=\eta\right) \\
& \quad-\mu_{q, \beta,\left(r_{1}, \ldots, r_{s}\right)}^{\mathbf{Z}^{d}, i}\left(Y(W)=\xi \mid Y\left(\mathbf{Z}^{d} \backslash W\right)=\eta^{\prime}\right) \mid \leqslant \varepsilon
\end{aligned}
$$

whenever $\eta, \eta^{\prime} \in S^{\mathbf{Z}^{d} \backslash W}$ are such that $\eta\left(\Lambda_{n} \backslash W\right)=\eta^{\prime}\left(\Lambda_{n} \backslash W\right)$. This we will prove by providing a coupling of two fuzzy spin configurations $Y$ and $Y^{\prime}$ whose respective distributions are given by the conditional distributions in (28), and such that $Y(W)=$ $Y^{\prime}(W)$ with probability at least $1-\varepsilon$. To this end, we will show that with high probability (at least $1-\varepsilon$ ) there is a (random) edge set $E$ in $\Lambda_{n} \backslash W$ which separates $W$ from $\mathbf{Z}^{d} \backslash \Lambda_{n}$ (in the sense that all paths from $W$ to $\mathbf{Z}^{d} \backslash \Lambda_{n}$ must pass through $E$ ) and which has the property that $U(E)=U^{\prime}(E) \equiv 0$, where $U$ and $U^{\prime}$ are random-cluster configurations corresponding to $Y$ and $Y^{\prime}$. We call such an $E$ a cutset, and it is in showing the existence (with high probability) of a cutset in our coupling that the condition on $\beta$ in Theorem 4.4(i) comes into play. Once we have such a cutset, we will be able to invoke Lemma 9.2 to guarantee that $Y$ and $Y^{\prime}$ are equal inside the cutset, and we will be done. The last step, however, requires special care since $E$ is a random edge set while Lemma 9.2 is formulated for a fixed edge set $E_{3}$.

With this outline-of-a-proof in mind, we now proceed towards a complete argument. To ensure that a cutset of the desired kind exists with sufficiently high probability, we 
will need some lemmas on stochastic domination. There is an abundance of stochastic domination results for the random-cluster model (see [15] or [20]); the following one is tailored particularly for our purposes.

Lemma 9.3. - Let $G=(V, E)$ and $G^{\prime}=\left(V^{\prime}, E^{\prime}\right)$ be finite or infinite graphs such that $G$ is a subgraph of $G^{\prime}$, with $V \subseteq V^{\prime}$ and

$$
E=\left\{e \in E^{\prime}: \text { both endpoints of } e \text { are in } V\right\} .
$$

Fix $p \in[0,1]$ and $q>q^{\prime} \geqslant 1$, let $\phi_{p, q}^{G}$ be a random-cluster measure for $G$ with parameters $p$ and $q$, and let $\phi_{p, q^{\prime}}^{G^{\prime}}$ be a random-cluster measure for $G^{\prime}$ with parameters $p$ and $q^{\prime}$. Let $F$ be an arbitrary finite subset of $E$, and let $\xi \in\{0,1\}^{E \backslash F}$ and $\xi^{\prime} \in$ $\{0,1\}^{E^{\prime} \backslash F}$ be edge configurations satisfying $\xi \preccurlyeq \xi^{\prime}(E \backslash F)$. Suppose $U \in\{0,1\}^{E}$ and $U^{\prime} \in\{0,1\}^{E^{\prime}}$ are picked according to $\phi_{p, q}^{G}$ and $\phi_{p, q^{\prime}}^{G^{\prime}}$, respectively. Write $\phi_{p, q}^{G} \mid \xi$ for the conditional distribution of $U(F)$ given $U(E \backslash F)=\xi$, and similarly let $\phi_{p, q^{\prime}}^{G^{\prime}} \mid \xi^{\prime}$ denote the conditional distribution of $U^{\prime}(F)$ given $U^{\prime}\left(E^{\prime} \backslash F\right)=\xi^{\prime}$. Then

$$
\phi_{p, q}^{G}\left|\xi \stackrel{\mathcal{D}}{\preccurlyeq} \phi_{p, q^{\prime}}^{G^{\prime}}\right| \xi^{\prime} .
$$

Proof. - This is a direct application of Holley's Theorem on stochastic domination (see [14, Theorem 4.8]), using just the single-edge conditional distributions (8) for random-cluster measures.

The point of Lemma 9.3 in this context is of course that it can be applied to the conditional distribution of the edge configuration $U\left(E^{Y, j}\right)$ on a monochromatic component $E^{Y, j}$ of the fuzzy spin configuration $Y$ on $G^{\prime}$. We need, however, to be able to handle all such components $E^{Y, 1}, \ldots, E^{Y, s}$ simultaneously, which requires a strengthening of Lemma 9.3. The next lemma provides such a strengthening, although specialized to the case $G^{\prime}=\mathbf{Z}^{d}$. We will also make the following additional assumption on the parameter values. The main result will then be proved under this assumption, and only in the end will we indicate how to adapt the proof to the case where the assumption fails.

Condition 9.4. - Suppose that the parameter values $\left(r_{1}, \ldots, r_{s}\right)$ for the fuzzy Potts model satisfy $r_{j} \geqslant 2$ for each $j$.

Lemma 9.5. - Consider the fuzzy Potts model on $\mathbf{Z}^{d}$ with parameter values as in Theorem 6.2, and let $(U, X, Y)$ and $\mathbf{P}$ be as in that theorem. Assume Condition 9.4. Let $F$ be an arbitrary finite subset of $E^{d}$. Then $\mathbf{P}$ admits conditional probabilities such that for any $\eta \in\{1, \ldots, s\}^{\mathbf{Z}^{d}}$ and any $\xi, \xi^{\prime} \in\{0,1\}^{E^{d} \backslash F}$ such that $\xi \preccurlyeq \xi^{\prime}$, we have that

the conditional distribution of $U(F)$ given that $Y=\eta$ and $U\left(E^{d} \backslash F\right)=\xi$ is stochastically dominated by

the conditional distribution of $U^{\prime}(F)$ given $U^{\prime}\left(E^{d} \backslash F\right)=\xi^{\prime}$, where $U^{\prime}$ is distributed according to a random-cluster measure for $\mathbf{Z}^{d}$ with parameters $p$ and $r_{1}$. 
Proof. - If we considered just $U\left(F \cap E^{Y, j}\right)$ for a monochromatic component $E^{Y, j}$ of the fuzzy spin configuration, then this would, due to Theorem 6.2 , be a direct application of Lemma 9.3; the conditions on $q$ of that result are fulfilled due to the convention (3) and Condition 9.4.

For the full result, we invoke Holley's Theorem [14, Theorem 4.8] using the singleedge conditional probabilities obtained in Theorem 6.2 and Remark 6.3.

An application of Lemma 9.5 is the following.

Lemma 9.6. - Consider the fuzzy Potts model on $\mathbf{Z}^{d}$ with parameter values as in Theorem 6.2, and let $(U, X, Y)$ and $\mathbf{P}$ be as in that theorem. Assume Condition 9.4. Then $\mathbf{P}$ admits conditional probabilities such that for any $\eta \in\{1, \ldots, s\}^{\mathbf{Z}^{d}}$,

$$
\text { the conditional distribution of } U \text { given } Y=\eta
$$

is stochastically dominated by

$$
\text { the wired random-cluster measure } \mu_{p, r_{1}}^{\mathbf{Z}^{d} \text {,wired }} \text {. }
$$

Proof. - Let $\Lambda_{n}, E_{n}, G_{n}$ and $\phi_{p, q^{\prime}}^{G_{n} \text {,wired }}$ be as in Section 6. The boundary condition used in $\phi_{p, q^{\prime}}^{G_{n} \text {,wired }}$ is tantamount to setting $Y(e)=1$ for all $e \in E^{d} \backslash E_{n}$, and we therefore have from Lemma 9.5 that the projection of $\phi_{p, q}^{G}$ on $E_{n} \cap E$ is stochastically dominated by the projection of $\phi_{p, q^{\prime}}^{G_{n} \text {,wired }}$ on $E_{n} \cap E$. Lemma 9.6 follows upon sending $n \rightarrow \infty$.

We are now equipped with the stochastic domination tools needed to go on with our proof of the main result.

Proof of Theorem 4.4(i) in the case where Condition 9.4 holds. - Fix an arbitrary finite vertex set $W \subset \mathbf{Z}^{d}$ and an $\varepsilon>0$; we need to show that (28) holds if $n$ is large enough.

Let $p=1-\mathrm{e}^{-2 \beta}$. By the assumption of the theorem that $\beta<\beta_{c}\left(d, r_{1}\right)$, we have that the corresponding random-cluster model is subcritical, i.e., that $\phi_{p, r_{1}}^{\mathbf{Z}^{d} \text {, wired }}$ assigns probability zero to the event that an infinite connected component of open edges exists. Hence,

$$
\lim _{n \rightarrow \infty} \phi_{p, r_{1}}^{\mathbf{Z}^{d} \text {,wired }}\left(\exists \text { an open path starting in } W \text { and ending in } \mathbf{Z}^{d} \backslash \Lambda_{n}\right)=0,
$$

so let us pick $n$ large enough so that this probability is at most $\varepsilon$.

Now let $\eta, \eta^{\prime} \in\{1, \ldots, s\}^{\mathbf{Z}^{d} \backslash W}$ be two arbitrary fuzzy spin configurations satisfying $\eta\left(\Lambda_{n} \backslash W\right)=\eta^{\prime}\left(\Lambda_{n} \backslash W\right)$. We shall construct a coupling of

(i) a triple $(U, X, Y)$ with distribution $\mathbf{P}$ conditioned on $Y\left(\mathbf{Z}^{d} \backslash W\right)=\eta$,

(ii) a triple $\left(U^{\prime}, X^{\prime}, Y^{\prime}\right)$ with distribution $\mathbf{P}$ conditioned on $Y^{\prime}\left(\mathbf{Z}^{d} \backslash W\right)=\eta^{\prime}$, and

(iii) an edge configuration $U^{\prime \prime} \in\{0,1\}^{E^{d}}$ with distribution $\phi_{p, r_{1}}^{\mathbf{Z}^{d}}$, wired .

We write $\mathbf{Q}$ for the probability measure underlying the coupling. If we can establish that $\mathbf{Q}\left(Y(W)=Y^{\prime}(W)\right) \geqslant 1-\varepsilon$, then (28) follows and the proof will be complete. The central objects in our coupling are therefore those in (i) and (ii); the edge configuration in (iii) is only there as a tool for keeping track of (i) and (ii). The coupling construction proceeds in several stages: first we determine the edge configurations $U, U^{\prime}$ and $U^{\prime \prime}$ 
outside of the box $\Lambda_{n}$, then we sequentially determine the statuses of the edges inside the box (one at a time in a certain random order to be described below), and finally we decide on the fuzzy spin variables in $W$. (We will not need to bother about specifying the true spin configurations $X$ and $X^{\prime}$.)

For the first stage of the coupling construction, note that Lemma 9.6 implies that we can pick $U\left(E^{d} \backslash E_{n}\right), U^{\prime}\left(E^{d} \backslash E_{n}\right)$, and $U^{\prime \prime}\left(E^{d} \backslash E_{n}\right)$ in such a way that

$$
\mathbf{Q}\left(U\left(E^{d} \backslash E_{n}\right) \preccurlyeq U^{\prime \prime}\left(E^{d} \backslash E_{n}\right) \quad \text { and } \quad U^{\prime}\left(E^{d} \backslash E_{n}\right) \preccurlyeq U^{\prime \prime}\left(E^{d} \backslash E_{n}\right)\right)=1,
$$

so let us do that.

The second stage is a kind of sequential technique which is related to the disagreement percolation technique of van den Berg and Maes [4], and (more closely) to the ideas of [17, proof of Lemma 3.4]. Fix an arbitrary enumeration $\left(e_{1}, \ldots, e_{m}\right)$ of all the edges in $E_{n}$. At each step $k$ of the sequential construction, select the edge $e_{l}$ with the smallest index $l$ satisfying

(a) $e_{l}$ has not been selected in any previous step, and

(b) $e_{l}$ is incident to some vertex in $\partial \Lambda_{n}$ or to some previously selected edge with $U^{\prime \prime}(e)=1$.

(If no such edge can be found - which is what we want! - then we go on to the third stage of the construction.) When an edge $e_{l}$ is selected, we also pick a random number $R_{l}$, uniformly distributed on $[0,1]$. Define $\alpha_{l}$ to be the conditional probability (under the measure $\mathbf{P}$ conditioned on $\left.Y\left(\mathbf{Z}^{d} \backslash W\right)=\eta\right)$, given everything about $(U, X, Y)$ that we have seen so far, that $U\left(e_{l}\right)=1$, and set

$$
U\left(e_{l}\right)= \begin{cases}1 & \text { if } R_{l}<\alpha_{l}, \\ 0 & \text { otherwise }\end{cases}
$$

Define $\alpha_{l}^{\prime}$ analogously, and set

$$
U^{\prime}\left(e_{l}\right)= \begin{cases}1 & \text { if } R_{l}<\alpha_{l}^{\prime} \\ 0 & \text { otherwise }\end{cases}
$$

Finally, set $\alpha_{l}^{\prime \prime}$ to be the conditional $\phi_{p, r_{i}}^{\mathbf{Z}^{d} \text {, wired }}$-probability that $U^{\prime \prime}\left(e_{l}\right)=1$ given what we have seen so far of $U^{\prime \prime}$, and set

$$
U^{\prime \prime}\left(e_{l}\right)= \begin{cases}1 & \text { if } R_{l}<\alpha_{l}^{\prime \prime}, \\ 0 & \text { otherwise }\end{cases}
$$

At stage $k=1$, Lemma 9.5 and (29) together imply that $\alpha_{l}<\alpha_{l}^{\prime \prime}$ and that $a_{l}^{\prime}<a_{l}^{\prime \prime}$. Hence, using (30), (31) and (32), we get that $U\left(e_{l}\right)<U^{\prime \prime}\left(e_{l}\right)$ and $U^{\prime}\left(e_{l}\right)<U^{\prime \prime}\left(e_{l}\right)$. By Lemma 9.5 and induction, we obtain the same thing at every stage $k$ of the sequential construction.

Suppose now that we have arrived at the situation where no edge $e \in\left(e_{1}, \ldots, e_{m}\right)$ satisfying (a) and (b) can be found. Write $E_{1}$ for the (random) set of edges whose values in $U, U^{\prime}$ and $U^{\prime \prime}$ have not been determined, set $V_{1}$ to be the set of vertices that are endpoints of edges in $E_{1}$, and define $G_{1}=\left(V_{1}, E_{1}\right)$. Also write $E_{3}$ for the set of edges 
whose values have been determined but which are incident to at least one edge in $E_{1}$. We must then have that $U^{\prime \prime}(e)=0$ for all $e \in E_{3}$ (because otherwise we would be able to continue the sequential construction). But then $U\left(E_{3}\right)=U^{\prime}\left(E_{3}\right) \equiv 0$ as well. Lemma 9.2 now guarantees that the conditional distribution of $\left(U\left(E_{1}\right), X\left(V_{1}\right), Y\left(V_{1}\right)\right)$ given what we have already seen of $(U, X, Y)$ is $\mathbf{P}^{G_{1}}$ conditioned on the event that $Y\left(V_{1} \backslash W\right)=$ $\eta\left(V_{1} \backslash W\right)$. The same applies to the conditional distribution of $\left(U^{\prime}\left(E_{1}\right), X^{\prime}\left(V_{1}\right), Y^{\prime}\left(V_{1}\right)\right)$ given what we have seen of $\left(U^{\prime}, X^{\prime}, Y^{\prime}\right)$. Hence we can take $\left(U\left(E_{1}\right), X\left(V_{1}\right), Y\left(V_{1}\right)\right)=$ $\left(U^{\prime}\left(E_{1}\right), X^{\prime}\left(V_{1}\right), Y^{\prime}\left(V_{1}\right)\right.$ in our coupling. In particular, we get $Y(W)=Y^{\prime}(W)$ if $W \subset$ $V_{1}$. Note now that if $W \nsubseteq V_{1}$, then $U^{\prime \prime}$ contains an open path starting in $W$ and ending in $\mathbf{Z}^{d} \backslash \Lambda_{n}$. Hence

$$
\mathbf{Q}\left(Y(W)=Y^{\prime}(W)\right) \geqslant \mathbf{Q}\left(W \subset V_{1}\right) \geqslant 1-\varepsilon
$$

by our choice of $n$, so the proof is complete.

It now only remains to remove Condition 9.4 from our assumptions.

Proof of Theorem 4.4(i) in the general case. - Note that if Condition 9.4 fails, i.e., if $r_{j}=1$ for some $j \in\{2, \ldots, s\}$, then Lemmas 9.5 and 9.6 fail. Hence, we cannot guarantee that $U\left(E_{3}\right)=U^{\prime}\left(E_{3}\right) \equiv 0$ in the cutset $E_{3}$ obtained in the above proof of Theorem 4.4(i).

But if we modify $(U, X, Y)$ into a triple $(\widetilde{U}, X, Y)$ by setting $(U, X, Y)=(\widetilde{U}, X, Y)$ except that $\widetilde{U}(e)=0$ for all edges $e=\langle x, y\rangle$ such that

$$
Y(x)=Y(y) \in\left\{j \in\{1, \ldots, s\}: r_{j}=1\right\},
$$

then it is easy to check that the lemmas will be true again.

A very important observation now is that the status $U(e)$ of such an edge, given that $Y(x)=Y(y) \in\left\{j \in\{1, \ldots, s\}: r_{j}=1\right\}$, is conditionally independent of everything else; this follows from Theorem 6.2 and Remark 6.3.

Let us now modify the sequential construction in the above proof of Theorem 4.4(i) as follows. Every time we determine the status of an edge $e=\langle x, y\rangle$ with $Y(x)=Y(y) \in$ $\left\{j \in\{1, \ldots, s\}: r_{j}=1\right\}$, we simply set $U(e)=U^{\prime}(e)=0$. The modified variants of Lemmas 9.5 and 9.6 will now guarantee that $U\left(E_{3}\right)=U^{\prime}\left(E_{3}\right) \equiv 0$ in the cutset $E_{3}$. The distribution of $U$ and $U^{\prime}$ in this coupling will of course not be the right ones, but the distributions of $Y$ and $Y^{\prime}$ will (due to the above observation about the conditional independence of the $U(e)$ 's). For this modified coupling, we will arrive at (33) in the same way as in the original version, and this completes the proof.

\section{Acknowledgement}

I thank the two referees for helpful suggestions and corrections.

\section{REFERENCES}

[1] M. Aizenman, J.T. Chayes, L. Chayes, C.M. Newman, Discontinuity of the magnetization in one-dimensional $1 /|x-y|^{2}$ Ising and Potts models, J. Stat. Phys. 50 (1988) 1-40. 
[2] M. Aizenman, H. Kesten, C.M. Newman, Uniqueness of the infinite cluster and continuity of connectivity functions for short- and long-range percolation, Comm. Math. Phys. 111 (1987) 505-532.

[3] D.J. Barsky, G.R. Grimmett, C.M. Newman, Percolation in half-spaces: equality of critical densities and continuity of the percolation probability, Probab. Theory Related Fields 90 (1991) 111-148.

[4] J. van den Berg, C. Maes, Disagreement percolation in the study of Markov fields, Ann. Probab. 22 (1994) 749-763.

[5] L. Chayes, Percolation and ferromagnetism on $\mathbf{Z}^{2}$ : the $q$-state Potts cases, Stochastic Process. Appl. 65 (1996) 209-216.

[6] A.C.D. van Enter, On the possible failure of the Gibbs property for measures on lattice systems, Markov Proc. Related Fields 2 (1996) 209-224.

[7] A.C.D. van Enter, R. Fernández, R. Kotecký, Pathological behavior of renormalizationgroup maps at high fields and above the transition temperature, J. Stat. Phys. 79 (1995) 969-992.

[8] A.C.D. van Enter, R. Fernández, A.D. Sokal, Regularity properties of position-space renormalization group transformations: Scope and limitations of Gibbsian theory, J. Stat. Phys. 72 (1993) 879-1167.

[9] A.C.D. van Enter, C. Maes, R.H. Schonmann, S. Shlosman, The Griffiths singularity random field, in: R. Minlos, Yu. Suhov, S. Shlosman (Eds.), On Dobrushin's Way. From Probability to Statistical Mechanics, American Mathematical Society, 2000, pp. 59-70.

[10] A.C.D. van Enter, C. Maes, S. Shlosman, Dobrushin's program on Gibbsianity restoration: weakly Gibbs and almost Gibbs random fields, in: R. Minlos, Yu. Suhov, S. Shlosman (Eds.), On Dobrushin's Way. From Probability to Statistical Mechanics, American Mathematical Society, 2000, pp. 51-58.

[11] R. Fernández, C.-E. Pfister, Global specifications and nonquasilocality of projections of Gibbs measures, Ann. Probab. 25 (1997) 1284-1315.

[12] C.M. Fortuin, P.W. Kasteleyn, On the random-cluster model. I. Introduction and relation to other models, Physica 57 (1972) 536-564.

[13] H.-O. Georgii, Gibbs Measures and Phase Transitions, de Gruyter, New York, 1988.

[14] H.-O. Georgii, O. Häggström, C. Maes, The random geometry of equilibrium phases, in: C. Domb, J.L. Lebowitz (Eds.), Phase Transitions and Critical Phenomena, Vol. 18, Academic Press, London, 2001, pp. 1-142.

[15] G.R. Grimmett, The stochastic random-cluster process, and the uniqueness of randomcluster measures, Ann. Probab. 23 (1995) 1461-1510.

[16] G.R. Grimmett, Percolation, 2nd Edition, Springer, New York, 1999.

[17] O. Häggström, Random-cluster representations in the study of phase transitions, Markov Proc. Related Fields 4 (1998) 275-321.

[18] O. Häggström, Positive correlations in the fuzzy Potts model, Ann. Appl. Probab. 9 (1999) 1149-1159.

[19] O. Häggström, Coloring percolation clusters at random, Stochastic Process. Appl. 96 (2001) 213-242.

[20] O. Häggström, J. Jonasson, R. Lyons, Coupling and Bernoullicity in random-cluster and Potts models, Bernoulli 8 (2002) 275-294.

[21] C. Külske, (Non-)Gibbsianness and phase transitions in random lattic spin models, Markov Proc. Related Fields 5 (1999) 357-383.

[22] C. Külske, Weakly Gibbsian representations for joint measures of quenched lattice spin models, Probab. Theory Related Fields 119 (2001) 1-30. 
[23] H. Künsch, S. Geman, A. Kehagias, Hidden Markov random fields, Ann. Appl. Probab. 5 (1995) 577-602.

[24] L. Laanait, A. Messager, J. Ruiz, Phase coexistence and surface tensions for the Potts model, Comm. Math. Phys. 105 (1986) 527-545.

[25] C. Maes, K. Vande Velde, The fuzzy Potts model, J. Phys. A 28 (1995) 4261-4271.

[26] C.M. Newman, L.S. Schulman, Infinite clusters in percolation models, J. Stat. Phys. 26 (1981) 613-628.

[27] A. Pisztora, Surface order large deviations for Ising, Potts and percolation models, Probab. Theory Related Fields 104 (1996) 427-466.

[28] R.H. Swendsen, J.-S. Wang, Nonuniversal critical dynamics in Monte Carlo simulations, Phys. Rev. Lett. 58 (1987) 86-88. 